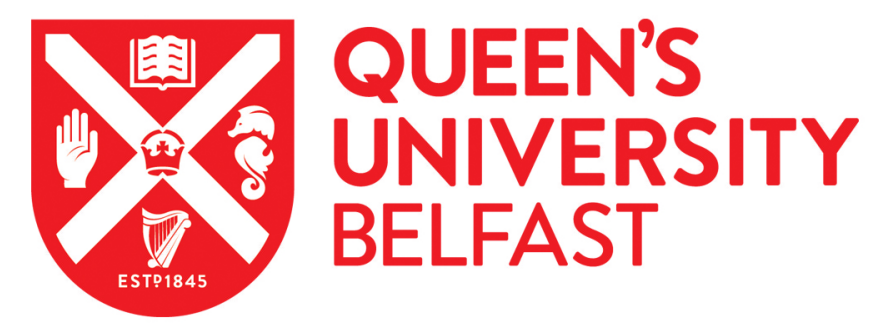

\title{
Beamforming Optimization for Physical Layer Security in MISO Wireless Networks
}

Sheng, Z., Tuan, H. D., Duong, Q., \& Poor, H. V. (2018). Beamforming Optimization for Physical Layer Security in MISO Wireless Networks. IEEE Transactions on Signal Processing, 66(14), 3710-3723.

https://doi.org/10.1109/TSP.2018.2835406

Published in:

IEEE Transactions on Signal Processing

Document Version:

Peer reviewed version

Queen's University Belfast - Research Portal:

Link to publication record in Queen's University Belfast Research Portal

Publisher rights

(c) 2018 IEEE. This work is made available online in accordance with the publisher's policies. Please refer to any applicable terms of use of the publisher.

\section{General rights}

Copyright for the publications made accessible via the Queen's University Belfast Research Portal is retained by the author(s) and / or other copyright owners and it is a condition of accessing these publications that users recognise and abide by the legal requirements associated with these rights.

Take down policy

The Research Portal is Queen's institutional repository that provides access to Queen's research output. Every effort has been made to ensure that content in the Research Portal does not infringe any person's rights, or applicable UK laws. If you discover content in the Research Portal that you believe breaches copyright or violates any law, please contact openaccess@qub.ac.uk. 


\title{
Beamforming Optimization for Physical Layer Security in MISO Wireless Networks
}

\author{
Zhichao Sheng, Hoang D. Tuan, Trung Q. Duong and H. Vincent Poor
}

\begin{abstract}
A wireless network of multiple transmitter-user pairs overheard by an eavesdropper, where the transmitters are equipped with multiple antennas while the users and eavesdropper are equipped with a single antenna, is considered. At different levels of wireless channel knowledge, the problem of interest is beamforming to optimize the users' quality-ofservice (QoS) in terms of their secrecy throughputs or maximize the network's energy efficiency under users' QoS. All these problems are seen as very difficult optimization problems with many nonconvex constraints and nonlinear equality constraints in beamforming vectors. The paper develops path-following computational procedures of low-complexity and rapid convergence for the optimal beamforming solution. Their practicability is demonstrated through numerical examples.
\end{abstract}

Index Terms-Multi-input single-output network, secure communication, energy-efficient communication, beamforming, pathfollowing algorithms.

\section{INTRODUCTION}

Securing information has emerged as one of the most critical issues in wireless communication [1], [2]. The broadcast nature of wireless transmissions implies that they can be quite vulnerable to adversary, who attempts to intercept their information delivery or overhear the confidential information intended for their users [3], [4]. Physical layer security (PLS) exploiting the physical properties of wireless channels [5], [6] has been proposed to ensure the secrecy of data transmissions to end-users of low complexity, for which encryption cannot be used. PLS is based on information theoretic characterizations of secrecy, under which the user secrecy throughput of a wireless transmission overheard by eavesdroppers (EVs) is determined as the difference between the user throughput and EVs' throughput [2], [7]. Transmit beamforming to improve the user throughput while controlling the throughput of the wiretapped signal at the EVs thus presents an effective way for secrecy throughput enhancement. Beamforming design for maximizing instantaneous secrecy throughput has been considered in [8]-[12] by semi-definite relaxation and randomization with the known inefficiency [13]. This beamforming design has been successfully addressed in [14], [15]. In regards to

This work was supported in part by the Australian Research Councils Discovery Projects under Project DP130104617, in part by the U.K. Royal Academy of Engineering Research Fellowship under Grant RF1415/14/22, and in part by the U.S. National Science Foundation under Grants CNS1702808 and ECCS-1647198

Z. Sheng and H. D. Tuan are with the School of Electrical and Data Engineering, University of Technology Sydney, Broadway, NSW 2007, Australia (email: zhichaosheng@163.com, Tuan.Hoang@uts.edu.au)

T. Q. Duong is with Queen's University Belfast, Belfast BT7 1NN, UK (email: trung.q.duong@qub.ac.uk)

H. V. Poor is with the Department of Electrical Engineering, Princeton University, Princeton, NJ 08544, USA (e-mail: poor@princeton.edu) outage probability, several works such as [16]-[19] used the Bernstein-type inequalities obtained in an unpublished work [20]. We will show that the results based on such Bernsteintype inequalities may be very conservative. Reference [21] considered outage region characterization of given beamformers under imperfect channel state information (CSI).

On the other hand, as energy efficiency (EE) became a very serious concern in wireless communication [22], [23], the secure energy efficiency (SEE), which is the ratio of the secrecy throughput to the total network power consumption, measured in terms of secrecy bits per Joule per Hertz is also increasingly important in PLS [24], [25]. Exploiting the perfect CSI, the SEE maximization in [26]-[28] is based on costly beamformers, which completely cancel the multiuser interference and wiretapped signal at the EVs. The computational complexity of the SEE optimization algorithms for single-user multi-input multi-output (MIMO)/single-input single-output (SISO) communications in [29] and [30] is also high as each iteration still involves a difficult nonconvex optimization problem. Our previous work [31] considered SEE optimization for a more general case of MIMO networks. SEE optimization was also considered in [15] for the worst case of uncertainties for users' and EVs' channels. There is no existing work on SEE optimization with secrecy throughput in terms of probability outage.

In this paper, we consider a network of multiple transmitteruser pairs overheard by an eavesdropper (EV). As the transmitters are assumed to be equipped with multiple antennas while the users and EV are equipped with a single antennas, the target is to design transmit beamformers to optimize either the users' quality-of-service (QoS) in terms of their secrecy throughput or the network's SEE under the users' QoS. It should be realized that these problems of beamforming design are still widely open for research, so we consider them at different levels of channel knowledge. The paper is structured as follows. Section II is devoted to the problem statements. Section III considers these problems under the perfect CSI of the all concerned channels, where path-following algorithms of low complexity are developed for their solution. In Section IV, the perfect CSI of the channels between the transmitters and user is assumed but only the distribution of the channels between the transmitters and EV is assumed known. As such, the EV's throughput is not deterministically defined but is defined through its probability outage, which leads to a nonlinear equation in beamforming vectors and the EV's throughput, making the beamforming designs much more computationally challenging. Under the same knowledge on the channels between the transmitters and EV in Section IV, Section V 
also assumes that the channels between the transmitters and users are uncertain with Gaussian distributed errors, under which there is no known result on the probability outage of the users' throughput. Nevertheless, based on a new result on outage probability obtained in Appendix I, both problems of users' QoS optimization and network's SEE optimization are successfully addressed. The simulation Section V shows the efficiency of the path-following algorithms developed in sections III-V. Conclusions are given in Section VI. Appendix I provides a new result on both upper bound and lower bound of the outage-aware user throughput. Appendix II shows the conservativeness of some other results, which are based on Bernstein type inequalities. Some fundamental deterministic inequalities that are used in Sections III-V are given in Appendix III.

Notation. The inner product between vectors $x$ and $y$ is defined as $\langle x, y\rangle=x^{H} y$. Analogously, $\langle X, Y\rangle=\operatorname{Trace}\left(X^{H} Y\right)$ for matrices $X$ and $Y$. Optimization variables are boldfaced. Also the notation $\sum_{j \neq i}^{M}$ refers to the summation taken over the index set $\{1, \ldots, M\} \backslash\{i\} . I$ is the identity matrix of appropriate dimension and $\mathcal{C N}(0, I)$ is the set of complex Gaussian random variables of zero means and identity covariance.

\section{Problem Statements}

Consider a communication network of $M$ transmitter-user pairs overheard by an EV. Each transmitter is equipped with $N_{t}$ transmit antennas while the users and EV are equipped by a single antenna. Thus, without the EV, the network looks very much like that considered in [32]-[36], where the channel knowledge is obtained from channel estimation, which is implemented at a central processing unit of the network, while beamforming is processed at a signal processing block of the network. The information $s_{i}$ for user $i$, which is normalized as $E\left(s_{i}^{2}\right)=1$, is beamformed by $\mathbf{w}_{i} \in \mathbb{C}^{N_{t}}$. The received signal at user $i$ is

$$
y_{i}=h_{i i}^{H} \mathbf{w}_{i} s_{i}+\sum_{j \neq i}^{M} h_{j i}^{H} \mathbf{w}_{j} s_{j}+n_{i},
$$

where $h_{j i} \in \mathbb{C}^{N_{t}}$ is the vector channel from transmitter $j$ to user $i$ and $n_{i}$ is the background noise with power $\sigma_{i}^{2}$.

Analogously, the received signal at the EV is

$$
y_{E}=\sum_{i=1}^{M} h_{i e}^{H} \mathbf{w}_{i} s_{i}+n_{e},
$$

where $h_{i e} \in \mathbb{C}^{N_{t}}$ is the vector channel from transmitter $i$ to the EV and $n_{e}$ is the background noise with power $\sigma_{e}^{2}$.

For $\mathbf{w} \triangleq\left[\mathbf{w}_{i}\right]_{i=1, \ldots, M}$, suppose that $f_{i}(\mathbf{w})$ is the throughput user $i$ while $g_{i}(\mathbf{w})$ is the wiretapped throughput for user $i$ at the EV. Our interest is the following optimization problems.

- Secrecy throughput maximin optimization under transmitters' power constraints:

$$
\begin{array}{r}
\max _{\boldsymbol{w}} \Phi(\mathbf{w}) \triangleq \min _{i=1, \ldots, M}\left[f_{i}(\mathbf{w})-g_{i}(\mathbf{w})\right] \quad \text { s.t. } \\
\left\|\mathbf{w}_{i}\right\|^{2} \leq P_{i}, i=1, \ldots, M,
\end{array}
$$

with $P_{i}$ given to set the limit of transmission power at transmitter $i$.
- Energy efficiency maximization over the secrecy throughput threshold constraints:

$$
\begin{array}{r}
\max _{\boldsymbol{w}} \Theta(\mathbf{w}) \triangleq\left[\sum_{i=1}^{M}\left[f_{i}(\mathbf{w})-g_{i}(\mathbf{w})\right]\right] / \pi(\mathbf{w}) \\
\text { s.t. } \quad(3 b), \\
f_{i}(\mathbf{w})-g_{i}(\mathbf{w}) \geq c_{i}, i=1, \ldots, M,
\end{array}
$$

with $c_{i}$ given to set the QoS threshold for user $i$ and the total network power consumption $\pi(\mathbf{w}) \triangleq \zeta \sum_{i=1}^{M}\left\|\mathbf{w}_{i}\right\|^{2}+$ $P_{c}$ in transmitting $\mathbf{w}_{i} s_{i}$, where $0<\zeta<1$ is the the reciprocal of the drain efficiency of the power amplifier and $P_{c}=\sum_{i=1}^{M} P_{c}^{i}$ with circuit power $P_{c}^{i}$ at transmitter $i$.

\section{INSTANTANEOUS SECRECY THROUGHPUT OPTIMIZATION}

When the perfect CSI of all channels is available at the transmitters, the user $i$ ' instantaneous throughput is defined by

$$
f_{i}(\mathbf{w}) \triangleq \ln \left(1+\frac{\left|h_{i i}^{H} \mathbf{w}_{i}\right|^{2}}{\sum_{j \neq i}\left|h_{j i}^{H} \mathbf{w}_{j}\right|^{2}+\sigma_{i}^{2}}\right),
$$

while the instantaneous wiretapped throughput for user $i$ at the $\mathrm{EV}$ is defined by

$$
g_{i}(\mathbf{w}) \triangleq \ln \left(1+\frac{\left|h_{i e}^{H} \mathbf{w}_{i}\right|^{2}}{\sum_{j \neq i}\left|h_{j e}^{H} \mathbf{w}_{j}\right|^{2}+\sigma_{e}^{2}}\right) .
$$

For $f_{i}(\mathbf{w})$ and $g_{i}(\mathbf{w})$ defined by (5) and (6), problem (3) is a particular case of the multi-cell beamforming design that considered in [14], [15]. We now propose a more efficient computation tailored for (3).

Let $w^{(\kappa)}$ be a feasible point for (3) found from $(\kappa-1)$ th iteration. Applying inequality (69) in the Appendix II for $x=$ $1 /\left|h_{i i}^{H} \mathbf{w}_{i}\right|^{2}, y=\sum_{j \neq i}^{M}\left|h_{j i}^{H} \mathbf{w}_{j}\right|^{2}+\sigma_{i}^{2}$, and $\bar{x}=1 /\left|h_{i i}^{H} w_{i}^{(\kappa)}\right|^{2}$, $\bar{y}=\sum_{j \neq i}^{M}\left|h_{j i}^{H} w_{j}^{(\kappa)}\right|^{2}+\sigma_{i}^{2}$ yields

$$
f_{i}(\mathbf{w}) \geq f_{i}^{(\kappa)}(\mathbf{w})
$$

for

$$
+\frac{x_{i}^{(\kappa)}}{1+x_{i}^{(\kappa)}}\left(2-\frac{f_{i}^{(\kappa)}(\mathbf{w})}{\ln \left(1+x_{i}^{(\kappa)}\right)} \triangleq\right.
$$

over the trust region

$$
\begin{array}{r}
2 \Re\left\{\left(w_{i}^{(\kappa)}\right)^{H} h_{i i} h_{i i}^{H} \mathbf{w}_{i}\right\}-\left|h_{i i}^{H} w_{i}^{(\kappa)}\right|^{2}>0, \\
i=1, \ldots, M,
\end{array}
$$

where

$$
x_{i}^{(\kappa)}=\frac{\left|h_{i i}^{H} w_{i}^{(\kappa)}\right|^{2}}{\sum_{j \neq i}^{M}\left|h_{j i}^{H} w_{j}^{(\kappa)}\right|^{2}+\sigma_{i}^{2}} .
$$


On the other hand, applying inequality (70) in the Appendix II for $x=\left|h_{i e}^{H} \mathbf{w}_{i}\right|^{2}, y=\sum_{j \neq i}^{M}\left|h_{j e}^{H} \mathbf{w}_{j}\right|^{2}+\sigma_{e}^{2}$ and $\bar{x}=$ $\left|h_{i e}^{H} w_{i}^{(\kappa)}\right|^{2}, \bar{y}=\sum_{j \neq i}^{M}\left|h_{j e}^{H} w_{j}^{(\kappa)}\right|^{2}+\sigma_{e}^{2}$ yields

$$
g_{i}(\mathbf{w}) \leq g_{i}^{(\kappa)}(\mathbf{w}),
$$

for

$$
\begin{aligned}
& g_{i}^{(\kappa)}(\boldsymbol{w}) \triangleq \\
& \ln \left(1+x_{e, i}^{(\kappa)}\right)+\frac{1}{1+x_{e, i}^{(\kappa)}} \\
& \times\left(\frac{\left|h_{i e}^{H} \mathbf{w}_{i}\right|^{2}}{\sum_{j \neq i}^{M}\left(2 \Re\left\{\left(w_{j}^{(\kappa)}\right)^{H} h_{j e} h_{j e}^{H} \mathbf{w}_{j}\right\}-\left|h_{j e}^{H} w_{j}^{(\kappa)}\right|^{2}\right)+\sigma_{e}^{2}}\right. \\
& \left.-x_{e, i}^{(\kappa)}\right)
\end{aligned}
$$

over the trust region

$$
\begin{array}{r}
\sum_{j \neq i}^{M}\left(2 \Re\left\{\left(w_{j}^{(\kappa)}\right)^{H} h_{j e} h_{j e}^{H} \mathbf{w}_{j}\right\}-\left|h_{j e}^{H} w_{j}^{(\kappa)}\right|^{2}\right)>0 \\
i=1, \ldots, M
\end{array}
$$

where

$$
x_{e, i}^{(\kappa)}=\left|h_{i e}^{H} w_{i}^{(\kappa)}\right|^{2} /\left(\sum_{j \neq i}^{M}\left|h_{j e}^{H} w_{j}^{(\kappa)}\right|^{2}+\sigma_{e}^{2}\right) .
$$

At the $\kappa$-th iteration we solve the following convex optimization problem to generate the next feasible point $w^{(\kappa+1)}$ :

$$
\begin{array}{r}
\max _{\boldsymbol{w}} \Phi^{(\kappa)}(\mathbf{w}) \triangleq \min _{i=1, \ldots, M}\left[f_{i}^{(\kappa)}(\boldsymbol{w})-g_{i}^{(\kappa)}(\boldsymbol{w})\right] \\
\text { s.t. } \quad(3 b),(9),(12) .
\end{array}
$$

The computational complexity of (13) is

$$
\mathcal{O}\left(n^{2} m^{2.5}+m^{3.5}\right),
$$

where $n=M N_{t}$ is the number of scalar variables, and $m=$ $3 M$ is the number of constraints.

From (7) and (10), it can be easily checked that $\Phi(\mathbf{w}) \geq$ $\Phi^{(\kappa)}(\mathbf{w}) \forall \mathbf{w}$ and $\Phi\left(w^{(\kappa)}\right)=\Phi^{(\kappa)}\left(w^{(\kappa)}\right)$. On the other hand, $\Phi^{(\kappa)}\left(w^{(\kappa+1)}\right)>\Phi^{(\kappa)}\left(w^{(\kappa)}\right)$ as far as $w^{(\kappa+1)} \neq w^{(\kappa)}$ because the former is the optimal solution of (13) while the latter is a feasible point for (13). We thus have the following chain of inequalities and equalities:

$$
\Phi\left(w^{(\kappa+1)}\right) \geq \Phi^{(\kappa)}\left(w^{(\kappa+1)}\right)>\Phi^{(\kappa)}\left(w^{(\kappa)}\right)=\Phi\left(w^{(\kappa)}\right),
$$

which implies that $w^{(\kappa+1)}$ is a better feasible point than $w^{(\kappa)}$ for the nonconvex optimization problem (3). Using a similar convergence argument as [37], we can show that at least the sequence $\left\{w^{(\kappa)}\right\}$ converges to its locally optimal solution. As such, the proposed Algorithm 1 a path-following computational procedure for (11).

Next, we address the EE maximization (4). A direct approach (see e.g. [15]) is based on a lower bounding approximation for the objective function in (4a). We now propose another approach, which uses the above approximation for the numerator of the objective function only, so the EE
Algorithm 1 Path-following algorithm for maximin instantaneous secrecy throughput optimization

Initialization: Set $\kappa=0$. Choose an initial feasible point $w^{(0)}$ for the convex constraints $(3 \mathrm{~b})$. Calculate $R_{\min }^{(0)}$ as the value of the objective in (3) at $w^{(0)}$. Set $\kappa=0$.

\section{repeat}

- Solve the convex optimization problem (13) to obtain the solution $w^{(\kappa+1)}$

- Calculate $R_{\min }^{(\kappa+1)}$ as the value of the objective in (3) at $w^{(\kappa+1)}$.

- Reset $\kappa+1 \rightarrow \kappa$.

until $\frac{\left.R_{\min }^{(\kappa+1)}-R_{\min }^{(\kappa)}\right)}{R_{\min }^{(\kappa)}} \leq \epsilon_{\mathrm{tol}}$.

maximization problem (4) is indeed not more computationally difficult than the throughput optimization problem (3).

As before, let $w^{(\kappa)}$ be its feasible point found from $(\kappa-1)$ th iteration. At the $\kappa$-th iteration, we solve the following convex optimization problem to generate the next feasible point $w^{(\kappa+1)}$ :

$$
\begin{array}{r}
\max _{\boldsymbol{w}} \sum_{i=1}^{M}\left[f_{i}^{(\kappa)}(\boldsymbol{w})-g_{i}^{(\kappa)}(\boldsymbol{w})\right]-\Theta\left(w^{(\kappa)}\right) \pi(\mathbf{w}) \\
\text { s.t. } \quad(3 b),(9),(12), \\
f_{i}^{(\kappa)}(\boldsymbol{w})-g_{i}^{(\kappa)}(\boldsymbol{w}) \geq c_{i}, i=1, \ldots, M .
\end{array}
$$

Note that $w^{(\kappa)}$ is a feasible point for (15), under which

$$
\sum_{i=1}^{M}\left[f_{i}^{(\kappa)}\left(w^{(\kappa)}\right)-g_{i}^{(\kappa)}\left(w^{(\kappa)}\right)\right]-\Theta\left(w^{(\kappa)}\right) \pi\left(w^{(\kappa)}\right)=0 .
$$

Therefore, as far as $w^{(\kappa+1)} \neq w^{(\kappa)}$, the optimal solution $w^{(\kappa+1)}$ of (15) must satisfy

$$
\begin{aligned}
& \sum_{i=1}^{M}\left[f_{i}^{(\kappa)}\left(w^{(\kappa+1)}\right)-g_{i}^{(\kappa)}\left(w^{(\kappa+1)}\right)\right]-\Theta\left(w^{(\kappa)}\right) \pi\left(w^{(\kappa+1)}\right)>0 \\
& \text { so } \\
& \begin{aligned}
\Theta\left(w^{(\kappa+1)}\right) & \triangleq \\
\sum_{i=1}^{M}\left[f_{i}\left(w^{(\kappa+1)}\right)-g_{i}\left(w^{(\kappa+1)}\right)\right] / \pi\left(w^{(\kappa+1)}\right) & \geq \\
\sum_{i=1}^{M}\left[f_{i}^{(\kappa)}\left(w^{(\kappa+1)}\right)-g_{i}^{(\kappa)}\left(w^{(\kappa+1)}\right)\right] / \pi\left(w^{(\kappa+1)}\right) & > \\
\Theta\left(w^{(\kappa)}\right), &
\end{aligned}
\end{aligned}
$$

implying that $w^{(\kappa+1)}$ is a better feasible point than $w^{(\kappa)}$ for the nonconvex optimization problem (4). As such, Algorithm 2, which is different from Algorithm 1 by solving the convex optimization problem (15) at the $\kappa$-th iteration to generate the next feasible point $w^{(\kappa+1)}$ instead of (13) in Algorithm 1, at least converges to a locally optimal solution.

A feasible point $w^{(0)}$ for (4) in the initialization of Algorithm 2 is found by using Algorithm 1 in solving the problem

$$
\max _{\mathbf{w}} \min _{i=1, \ldots, M}\left[f_{i}(\mathbf{w})-g_{i}\left(\mathbf{w}_{i}\right)\right] / c_{i} \quad \text { s.t. } \quad(3 b) .
$$

Namely Algorithm 1 will terminate whenever $\min _{i=1, \ldots, M}\left[f_{i}\left(w^{(\kappa)}\right)-g_{i}\left(w^{(\kappa)}\right)\right] / c_{i} \geq 1$. 


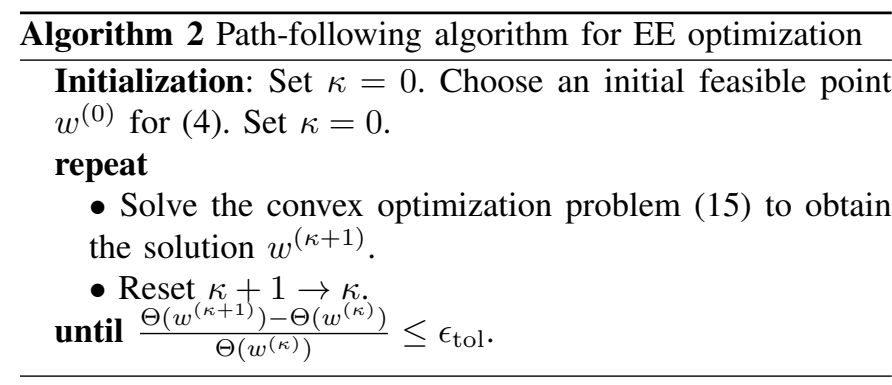

\section{EV'S OUTAGE PROBABILITY MAXIMIZATION}

When the EV is no longer part of the legitimate network, the assumption on the perfect CSI for the wiretapped channels $h_{j e}$ at the transmitters made in the previous section is not practical. Instead, it is common to assume that only the wiretapped channel distribution [7]

$$
h_{j e}=\sqrt{\bar{h}_{j e}} \chi_{j}, \chi_{j} \in \mathcal{C N}(0, I), j=1, \ldots, M
$$

is known, where $\sqrt{\bar{h}_{j e}}$ is a deterministic quantity which is usually dependent on the distance from the transmitter $j$ to the EV. The user throughput $f_{i}(\mathbf{w})$ is still defined by (5) but the wiretapped throughput $g_{i}(\mathbf{w})$ for user $i$ at the EV is defined via the following outage probability instead of the instantaneous throughput defined by (6):

$$
\begin{array}{r}
\max \left\{\ln \left(1+\mathbf{r}_{i}\right): \operatorname{Prob}\left(\frac{\bar{h}_{i e}\left|\chi_{i}^{H} \mathbf{w}_{i}\right|^{2}}{\sum_{j \neq i}^{M} \bar{h}_{j e}\left|\chi_{j}^{H} \mathbf{w}_{j}\right|^{2}+\sigma_{e}^{2}}\right.\right. \\
\left.\left.<\mathbf{r}_{i}\right)<\epsilon_{E V}\right\}
\end{array}
$$

for $\epsilon_{E V}>0$. Note that $\left|\chi_{j}^{H} \mathbf{w}_{j}\right|^{2}$ is an exponential distribution with mean $\left\|\mathbf{w}_{j}\right\|^{2}$. Therefore, by [38], this throughput is $\ln (1+$ $\mathbf{r}_{i}$ ), where

$$
g_{i, o}\left(\mathbf{w}, \mathbf{r}_{i}\right)=0
$$

for

$$
\begin{aligned}
g_{i, o}\left(\mathbf{w}, \mathbf{r}_{i}\right) \triangleq & \bar{h}_{i e} \ln \left(1-\epsilon_{E V}\right)+\sigma_{e}^{2} \frac{\mathbf{r}_{i}}{\left\|\mathbf{w}_{i}\right\|^{2}} \\
& +\bar{h}_{i e} \sum_{j \neq i}^{M} \ln \left(1+\frac{\mathbf{r}_{i} \bar{h}_{j e}\left\|\mathbf{w}_{j}\right\|^{2}}{\bar{h}_{i e}\left\|\mathbf{w}_{i}\right\|^{2}}\right),
\end{aligned}
$$

which increases in $\mathbf{r}_{i}$ with $\mathbf{w}$ held fixed.

Similarly to [38, Prop. 1] the problem of secrecy rate maximin optimization (3) is equivalently formulated by

$$
\begin{array}{r}
\max _{\boldsymbol{w}, \mathbf{r}} \min _{i=1, \ldots, M}\left[\ln \left(1+\frac{\left|h_{i i}^{H} \mathbf{w}_{i}\right|^{2}}{\sum_{j \neq i}\left|h_{j i}^{H} \mathbf{w}_{j}\right|^{2}+\sigma_{i}^{2}}\right)\right. \\
\left.-\ln \left(1+\mathbf{r}_{i}\right)\right] \text { s.t } \quad(3 b), \\
g_{i, o}\left(\mathbf{w}, \mathbf{r}_{i}\right) \geq 0, i=1, \ldots, M, \\
\mathbf{r}_{i}>0,
\end{array}
$$

where the nonlinear equality constraint in (19) has been replaced by the nonconvex constraint (21b).

The main difficulty is to develop a lower bounding approximation for the function $g_{i, o}\left(\mathbf{w}, \mathbf{r}_{i}\right)$ at a feasible point $\left(w^{(\kappa)}, r^{(\kappa)}\right)$ for $(21)$, which is found from $(\kappa-1)$ th iteration. Applying inequality (69) for $x=1 / \mathbf{r}_{i} \bar{h}_{j e}\left\|\mathbf{w}_{j}\right\|^{2}$, $y=\bar{h}_{i e}\left\|\mathbf{w}_{i}\right\|^{2}$, and $\bar{x}=1 / r_{i}^{(\kappa)} \bar{h}_{j e}\left\|w_{j}^{(\kappa)}\right\|^{2}, \bar{y}=\bar{h}_{i e}\left\|w_{i}^{(\kappa)}\right\|^{2}$ yields

$$
\ln \left(1+\frac{\mathbf{r}_{i} \bar{h}_{j e}\left\|\mathbf{w}_{j}\right\|^{2}}{\bar{h}_{i e}\left\|\mathbf{w}_{i}\right\|^{2}}\right) \geq \lambda_{i j}^{(\kappa)}\left(\mathbf{r}_{i}, \mathbf{w}_{j}, \mathbf{w}_{i}\right)
$$

over the trust region

$$
2 \Re\left\{\left(w_{j}^{(\kappa)}\right)^{H} \mathbf{w}_{j}\right\}-\left\|w_{j}^{(\kappa)}\right\|^{2}>0
$$

for

$$
\begin{aligned}
\lambda_{i j}^{(\kappa)}\left(\mathbf{r}_{i}, \mathbf{w}_{j}, \mathbf{w}_{i}\right) & \triangleq \\
\ln \left(1+x_{i j}^{(\kappa)}\right) & \\
+y_{i j}^{(\kappa)}\left(2-\frac{r_{i}^{(\kappa)} \bar{h}_{j e}\left\|w_{j}^{(\kappa)}\right\|^{2}}{\mathbf{r}_{i} \bar{h}_{j e}\left(2 \Re\left\{\left(w_{j}^{(\kappa)}\right)^{H} \mathbf{w}_{j}\right\}-\left\|w_{j}^{(\kappa)}\right\|^{2}\right)}\right. & \bar{h}_{i e}\left\|\mathbf{w}_{i}\right\|^{2} \\
-\frac{\ln \left(1+x_{i j}^{(\kappa)}\right)}{\bar{h}_{i e}\left\|w_{i}^{(\kappa)}\right\|^{2}} & = \\
+y_{i j}^{(\kappa)}\left(2-\frac{r_{i}^{(\kappa)}\left\|w_{j}^{(\kappa)}\right\|^{2}}{\mathbf{r}_{i}\left(2 \Re\left\{\left(w_{j}^{(\kappa)}\right)^{H} \mathbf{w}_{j}\right\}-\left\|w_{j}^{(\kappa)}\right\|^{2}\right)}\right. & \left\|\mathbf{w}_{i}\right\|^{2} \\
-\frac{w_{i}^{(\kappa)} \|^{2}}{n} &
\end{aligned}
$$

and $x_{i j}^{(\kappa)} \triangleq r_{i}^{(\kappa)} \bar{h}_{j e}\left\|w_{j}^{(\kappa)}\right\|^{2} / \bar{h}_{i e}\left\|w_{i}^{(\kappa)}\right\|^{2}$ and $y_{i j}^{(\kappa)} \triangleq$ $x_{i j}^{(\kappa)} /\left(x_{i j}^{(\kappa)}+1\right)$.

Furthermore, applying inequality (72) in the Appendix yields

$$
\frac{\mathbf{r}_{i}}{\left\|\mathbf{w}_{i}\right\|^{2}} \geq \beta_{i}^{(\kappa)}\left(\mathbf{r}_{i}, \mathbf{w}_{i}\right)
$$

where

$$
\beta_{i}^{(\kappa)}\left(\mathbf{r}_{i}, \mathbf{w}_{i}\right) \triangleq 2 \frac{\sqrt{r_{i}^{(\kappa)}}}{\left\|w_{i}^{(\kappa)}\right\|^{2}} \sqrt{\mathbf{r}_{i}}-\frac{r_{i}^{(\kappa)}}{\left\|w_{i}^{(\kappa)}\right\|^{4}}\left\|\mathbf{w}_{i}\right\|^{2},
$$

which is a concave function.

Based on (22) and (25) we obtain

$$
g_{i, o}\left(\mathbf{w}, \mathbf{r}_{i}\right) \geq g_{i, o}^{(\kappa)}\left(\mathbf{w}, \mathbf{r}_{i}\right)
$$

for

$$
\begin{aligned}
g_{i, o}^{(\kappa)}\left(\mathbf{w}, \mathbf{r}_{i}\right) \triangleq & \bar{h}_{i e} \ln \left(1-\epsilon_{E V}\right)+\sigma_{e}^{2} \beta_{i}^{(\kappa)}\left(\mathbf{r}_{i}, \mathbf{w}_{i}\right) \\
& +\bar{h}_{i e} \sum_{j \neq i}^{M} \lambda_{i j}^{(\kappa)}\left(\mathbf{r}_{i}, \mathbf{w}_{j}, \mathbf{w}_{i}\right),
\end{aligned}
$$

which is a concave function satisfying

$$
g_{i, o}\left(w^{(\kappa)}, r_{i}^{(\kappa)}\right)=g_{i, o}^{(\kappa)}\left(w^{(\kappa)}, r_{i}^{(\kappa)}\right) .
$$

Also, following [38], the second term in the objective (21a) is upper bounded by the linear function

$$
a_{i}^{(\kappa)}\left(\mathbf{r}_{i}\right)=\ln \left(1+r_{i}^{(\kappa)}\right)-\frac{r_{i}^{(\kappa)}}{r_{i}^{(\kappa)}+1}+\frac{\mathbf{r}_{i}}{r_{i}^{(\kappa)}+1},
$$


while the first term in (21a) is lower bounded by $f_{i}^{(\kappa)}(\boldsymbol{w})$ defined by (8) over the trust region (9).

We solve the following convex program at the $\kappa$-th iteration to generate the next feasible point $\left(w^{(\kappa+1)}, r_{u}^{(\kappa+1)}\right)$ :

$$
\begin{array}{r}
\max _{\boldsymbol{w}, \mathbf{r}} \min _{i=1, \ldots, M}\left[f_{i}^{(\kappa)}(\mathbf{w})-a_{i}^{(\kappa)}\left(\mathbf{r}_{i}\right)\right] \\
\text { s.t } \quad(3 b),(9),(21 c),(23), \\
g_{i, o}^{(\kappa)}\left(\mathbf{w}, \mathbf{r}_{i}\right) \geq 0, i=1, \ldots, M .
\end{array}
$$

The computational complexity of (30) is (14) for $n=M N_{t}+$ $M$ and $m=5 M$.

Then, $r_{i}^{(\kappa+1)}$ is found from solving the nonlinear equation

$$
\psi_{i}\left(\mathbf{r}_{i}\right) \triangleq g_{i, o}\left(w^{(\kappa)}, \mathbf{r}_{i}\right)=0, \quad i=1, \ldots, M
$$

by bisection on $\left[0, r_{u, i}^{(\kappa+1)}\right]$ with tolerance $\epsilon_{b}$ such that

$$
0 \leq \psi_{i}\left(r_{i}^{(\kappa+1)}\right) \leq \epsilon_{b}
$$

A bisection on $\left[r_{l}, r_{u}\right]$ for solving $\psi_{i}\left(\mathbf{r}_{i}\right)=0$ where $\psi_{i}$ increases in $\mathbf{r}_{\mathbf{i}}>0$ is implemented as follows:

- Define $r_{i}=\left(r_{l}+r_{u}\right) / 2$. Reset $r_{l}=r_{i}$ if $\psi_{i}\left(r_{i}\right)<0$. Otherwise reset $r_{u}=r_{i}$.

- Terminate until $0 \leq \psi_{i}\left(r_{i}\right) \leq \epsilon_{b}$.

Like Algorithm 1, Algorithm 3 at least converges to a locally optimal solution of (21).

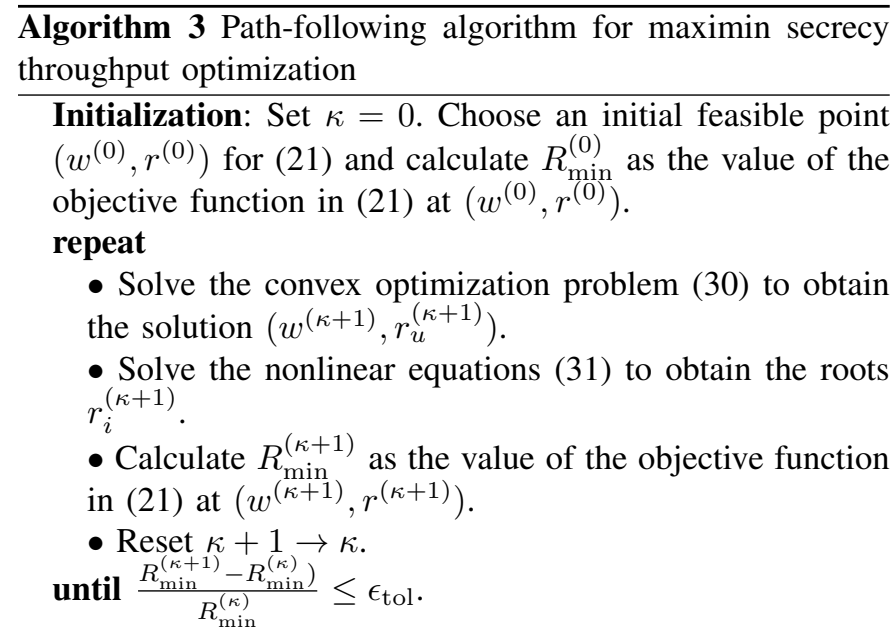

Next, the SEE maximization problem (4) can be formulated as

$$
\begin{array}{r}
\max _{\boldsymbol{w}, \mathbf{r}} \Theta(\boldsymbol{w}, \mathbf{r}) \triangleq \sum_{i=1}^{M}\left[\ln \left(1+\frac{\left|h_{i i}^{H} \mathbf{w}_{i}\right|^{2}}{\sum_{j \neq i}\left|h_{j i}^{H} \mathbf{w}_{j}\right|^{2}+\sigma_{i}^{2}}\right)\right. \\
\left.-\ln \left(1+\mathbf{r}_{i}\right)\right] / \pi(\mathbf{w}) \quad \text { s.t } \quad(3 b),(21 b),(21 c), \\
\ln \left(1+\frac{\left|h_{i i}^{H} \mathbf{w}_{i}\right|^{2}}{\sum_{j \neq i}\left|h_{j i}^{H} \mathbf{w}_{j}\right|^{2}+\sigma_{i}^{2}}\right)-\ln \left(1+\mathbf{r}_{i}\right) \geq c_{i}, \\
i=1, \ldots, M,
\end{array}
$$

where like (3b), $c_{i}$ in (33b) set the QoS threshold for user $i$.

As such, (33) is addressed by the following iterations with the convergence guaranteed.
- Initialization. Use Algorithm 3 to obtain a feasible point $\left(w^{(0)}, r^{(0)}\right)$ and define

$$
\theta^{(0)} \triangleq \sum_{i=1}^{M}\left[f_{i}\left(w^{(0)}\right)-\ln \left(1+r_{i}^{(0)}\right)\right] / \pi\left(w^{(0)}\right) .
$$

- $\kappa$-th iteration. Let $\left(w^{(\kappa)}, r^{(\kappa)}\right)$ be a feasible point found from the $(\kappa-1)$ th iteration. Define

$$
\theta^{(\kappa)} \triangleq \sum_{i=1}^{M}\left[f_{i}\left(w^{(\kappa)}\right)-\ln \left(1+r_{i}^{(\kappa)}\right)\right] / \pi\left(w^{(\kappa)}\right)
$$

and then solve the following convex optimization problem to generate the next feasible point $\left(w^{(\kappa+1)}, r_{u}^{(\kappa+1)}\right)$ :

$$
\begin{array}{r}
\max _{\boldsymbol{w}, \mathbf{r}} \sum_{i=1}^{M}\left[f_{i}^{(\kappa)}(\mathbf{w})-a_{i}^{(\kappa)}\left(\mathbf{r}_{i}\right)\right]-\Theta\left(w^{(\kappa)}, r^{(\kappa)}\right) \pi(\mathbf{w}) \\
\text { s.t. } \quad(3 b),(9),(21 c),(23),(30 b), \\
f_{i}^{(\kappa)}(\mathbf{w})-a_{i}^{(\kappa)}\left(\mathbf{r}_{i}\right) \geq c_{i}, i=1, \ldots M .
\end{array}
$$

Further, $r_{i}^{(\kappa+1)}$ is found from solving (31).

\section{ROBUST OPTIMIZATION TO COMPENSATE USERS' OUTAGE PROBABILITY}

Now assume that the wiretapped channel $h_{j e}$ is in form (17), so the wiretapped throughput $g_{i}(\mathbf{w})$ for user $i$ at the EV is defined via (18) but

$$
h_{j i}=\bar{h}_{j i}+\delta \chi_{j i}
$$

for $\chi_{j i} \in \mathcal{C N}(0, I)$ and $0<\delta<<1$. The term $\delta \chi_{j i}$ thus represents the channel error in channel state estimation. Then the user $i$ 's throughput $f_{i}(\mathbf{w})$ is implicitly defined through the outage probability as

$$
\begin{aligned}
& \varphi_{i, o}(\mathbf{w}) \triangleq \\
& \max \left\{\ln \left(1+\mathbf{R}_{i}\right):\right. \\
& \operatorname{Prob}\left(\frac{\left|\bar{h}_{i i}^{H} \mathbf{w}_{i}\right|^{2}}{\delta\left|\chi_{i i}^{H} \mathbf{w}_{i}\right|^{2}+\sum_{j \neq i}^{M}\left|\left(\bar{h}_{j i}+\delta \chi_{j i}\right)^{H} \mathbf{w}_{j}\right|^{2}+\sigma_{i}^{2}}\right. \\
& \left.\left.<\mathbf{R}_{i}\right)<\epsilon\right\}
\end{aligned}
$$

for $\epsilon>0$.

Note that [39]

$$
\begin{aligned}
& \left|\left(\bar{h}_{j i}+\delta \chi_{j i}\right)^{H} \mathbf{w}_{j}\right|^{2} \\
& \left|\left((1-\delta) \bar{h}_{j i} /(1-\delta)+\delta \chi_{j i}\right)^{H} \mathbf{w}_{j}\right|^{2} \leq \\
& (1-\delta)^{-1}\left|\bar{h}_{j i} \mathbf{w}_{j}\right|^{2}+\delta\left|\chi_{j i}^{H} \mathbf{w}_{j}\right|^{2},
\end{aligned}
$$

which implies

$$
\frac{\left|\bar{h}_{i i}^{H} \mathbf{w}_{i}\right|^{2}}{\delta\left|\chi_{i i}^{H} \mathbf{w}_{i}\right|^{2}+\sum_{j \neq i}^{M}\left|\left(\bar{h}_{j i}+\delta \chi_{j i}\right)^{H} \mathbf{w}_{j}\right|^{2}+\sigma_{i}^{2}} \quad \geq
$$


Consequently,

$$
\begin{aligned}
& \operatorname{Prob}\left(\frac{\left|\bar{h}_{i i}^{H} \mathbf{w}_{i}\right|^{2}}{\delta\left|\chi_{i i}^{H} \mathbf{w}_{i}\right|^{2}+\sum_{j \neq i}^{M}\left|\left(\bar{h}_{j i}+\delta \chi_{j i}\right)^{H} \mathbf{w}_{j}\right|^{2}+\sigma_{i}^{2}}\right. \\
&\left.<\mathbf{R}_{i}\right) \leq
\end{aligned}
$$

$$
\begin{aligned}
& \operatorname{Prob}\left(\frac{\left|\bar{h}_{i i}^{H} \mathbf{w}_{i}\right|^{2}}{(1-\delta)^{-1} \sum_{j \neq i}^{M}\left|\bar{h}_{j i}^{H} \mathbf{w}_{j}\right|^{2}+\delta \sum_{j=1}^{M}\left|\chi_{j i}^{H} \mathbf{w}_{j}\right|^{2}+\sigma_{i}^{2}}\right. \\
& \left.<\mathbf{R}_{i}\right)
\end{aligned}
$$

Proposition 1: It is true that

$$
\begin{aligned}
& \varphi_{i, o}(\mathbf{w}) \geq \\
& \bar{\varphi}_{i, o}(\mathbf{w}) \triangleq \\
& \max \left\{\ln \left(1+\mathbf{R}_{i}\right):\right. \\
& \operatorname{Prob}\left(\frac{\left|\bar{h}_{i i}^{H} \mathbf{w}_{i}\right|^{2}}{(1-\delta)^{-1} \sum_{j \neq i}^{M}\left|\bar{h}_{j i}^{H} \mathbf{w}_{j}\right|^{2}+\delta \sum_{j=1}^{M}\left|\chi_{j i}^{H} \mathbf{w}_{j}\right|^{2}+\sigma_{i}^{2}}\right. \\
& \left.\left.<\mathbf{R}_{i}\right)<\epsilon\right\} .
\end{aligned}
$$

Proof: By (37), if $\mathbf{R}_{i}>0$ such that

$$
\begin{aligned}
& \operatorname{Prob}\left(\frac{\left|\bar{h}_{i i}^{H} \mathbf{w}_{i}\right|^{2}}{(1-\delta)^{-1} \sum_{j \neq i}^{M}\left|\bar{h}_{j i}^{H} \mathbf{w}_{j}\right|^{2}+\delta \sum_{j=1}^{M}\left|\chi_{j i}^{H} \mathbf{w}_{j}\right|^{2}+\sigma_{i}^{2}}\right. \\
& \left.<\mathbf{R}_{i}\right)<\epsilon
\end{aligned}
$$

then

$$
\begin{aligned}
& \operatorname{Prob}\left(\frac{\left|\bar{h}_{i i}^{H} \mathbf{w}_{i}\right|^{2}}{\delta\left|\chi_{i i}^{H} \mathbf{w}_{i}\right|^{2}+\sum_{j \neq i}^{M}\left|\left(\bar{h}_{j i}+\delta \chi_{j i}\right)^{H} \mathbf{w}_{j}\right|^{2}+\sigma_{i}^{2}}\right. \\
& \left.<\mathbf{R}_{i}\right)<\epsilon
\end{aligned}
$$

and (38) follows.

Applying (64) in Appendix I for

$$
a=\left|\bar{h}_{i i}^{H} \mathbf{w}_{i}\right|^{2}, b=(1-\delta)^{-1} \sum_{j \neq i}^{M}\left|\bar{h}_{j i}^{H} \mathbf{w}_{j}\right|^{2}+\sigma_{i}^{2}
$$

gives

$$
\begin{array}{r}
\varphi_{i, o}(\mathbf{w}) \geq \\
\max \left\{\ln \left(1+\mathbf{R}_{i}\right): \delta\left[\delta_{M}\left\|\mathbf{w}_{\text {min }}\right\|^{2}\right.\right. \\
\left.\left.+\frac{M-1}{2}\left\|\mathbf{w}_{\text {min }}\right\|^{2} \ln \frac{\varphi_{i}\left(\mathbf{w}, \mathbf{R}_{i}\right)}{\left\|\mathbf{w}_{\text {min }}\right\|^{2}}\right] \leq \varphi_{i}\left(\mathbf{w}, \mathbf{R}_{i}\right)\right\},
\end{array}
$$

where

$$
\begin{aligned}
\varphi_{i}\left(\mathbf{w}, \mathbf{R}_{i}\right) \triangleq & \left|\bar{h}_{i i}^{H} \mathbf{w}_{i}\right|^{2} \\
\mathbf{R}_{i} & {\left[(1-\delta)^{-1} \sum_{j \neq i}^{M}\left|\bar{h}_{j i}^{H} \mathbf{w}_{j}\right|^{2}+\sigma_{i}^{2}\right], } \\
0<\delta_{M} \triangleq & -\left(\ln \epsilon-\ln M+\frac{1}{M} \sum_{i=1}^{M} \ln \Gamma(i)\right. \\
& \left.+\frac{M-1}{2} \ln \delta\right) \\
= & \ln \epsilon^{-1}+\ln M-\frac{1}{M} \sum_{i=1}^{M} \ln \Gamma(i) \\
& +\frac{M-1}{2} \ln \delta^{-1}, \\
\text { and } \quad & \left\|\mathbf{w}_{\min }\right\|^{2}=\min _{i=1, \ldots, M}\left\|\mathbf{w}_{i}\right\|^{2} .
\end{aligned}
$$

Recall that $\Gamma(i)$ are defined from (64).

Therefore, the problem of secrecy rate maximin optimization (3) is formulated by

$$
\begin{array}{r}
\max _{\boldsymbol{w}, \mathbf{R}, \mathbf{r}} \min _{i=1, \ldots, M}\left[\ln \left(1+\mathbf{R}_{i}\right)-\ln \left(1+\mathbf{r}_{i}\right)\right] \\
\text { s.t } \quad(3 b),(21 b),(21 c), \\
\varphi_{i}\left(\mathbf{w}, \mathbf{R}_{i}\right)>0, i=1, \ldots, M, \\
\delta\left[\delta_{M}\left\|\mathbf{w}_{\min }\right\|^{2}+\frac{M-1}{2}\left\|\mathbf{w}_{\min }\right\|^{2} \ln \frac{\varphi_{i}\left(\mathbf{w}, \mathbf{R}_{i}\right)}{\left\|\mathbf{w}_{\min }\right\|^{2}}\right] \\
\leq \varphi_{i}\left(\mathbf{w}, \mathbf{R}_{i}\right), i=1, \ldots, M,
\end{array}
$$

where $\ln \left(1+\mathbf{R}_{i}\right)-\ln \left(1+\mathbf{r}_{i}\right)$ in (40a) represents a lower bound for the user $i$ 's secrecy throughput.

Constraints (21b), (40b)-(40c) in (40) are nonconvex, which need to be innerly approximated at each iteration. Let $\left(w^{(\kappa)}, R^{(\kappa)}, r^{(\kappa)}\right)$ be a feasible point for (40) found from the $(\kappa-1)$ th iteration. We have provided an inner approximation for (21b) by (23) and (30b). Note that $\left|\bar{h}_{i i}^{H} \mathbf{w}_{i}\right|^{2} / \mathbf{R}_{i}$ is a convex function, so

$$
\left|\bar{h}_{i i}^{H} \mathbf{w}_{i}\right|^{2} / \mathbf{R}_{i} \geq \ell_{i}^{(\kappa)}\left(\mathbf{w}_{i}, \mathbf{R}_{i}\right)
$$

for

$$
\begin{aligned}
\ell_{i}^{(\kappa)}\left(\mathbf{w}_{i}, \mathbf{R}_{i}\right) \triangleq & 2 \Re\left\{\left(w_{i}^{(\kappa)}\right)^{H} \bar{h}_{i i} \bar{h}_{i i}^{H} \mathbf{w}_{i}\right\} / R_{i}^{(\kappa)} \\
& -\mathbf{R}_{i}\left|\bar{h}_{i i}^{H} w_{i}^{(\kappa)}\right|^{2} /\left(R_{i}^{(\kappa)}\right)^{2},
\end{aligned}
$$

which is the linearization of $\left|\bar{h}_{i i}^{H} \mathbf{w}_{i}\right|^{2} / \mathbf{R}_{i}$ at $\left(w_{i}^{(\kappa)}, R_{i}^{(\kappa)}\right)$. Therefore, the nonconvex constraint (40b) is innerly approximated by the convex constraint

$$
\begin{array}{r}
\ell_{i}^{(\kappa)}\left(\mathbf{w}_{i}, \mathbf{R}_{i}\right)>(1-\delta)^{-1} \sum_{j \neq i}^{M}\left|\bar{h}_{j i}^{H} \mathbf{w}_{j}\right|^{2}+\sigma_{i}^{2}, \\
i=1, \ldots, M .
\end{array}
$$

Furthermore, for

it is true that

$$
x_{i}^{(\kappa)} \triangleq \frac{\varphi_{i}\left(w^{(\kappa)}, R_{i}^{(\kappa)}\right)}{\left\|w_{\min }^{(\kappa)}\right\|^{2}}
$$

$$
\ln \frac{\varphi_{i}\left(\mathbf{w}, \mathbf{R}_{i}\right)}{\left\|\mathbf{w}_{\min }\right\|^{2}} \leq \ln \left(x_{i}^{(\kappa)}\right)-1+\frac{\varphi_{i}\left(\mathbf{w}, \mathbf{R}_{i}\right)}{x_{i}^{(\kappa)}\left\|\mathbf{w}_{\min }\right\|^{2}}
$$


that yields

$\left\|\mathbf{w}_{\min }\right\|^{2} \ln \frac{\varphi_{i}\left(\mathbf{w}, \mathbf{R}_{i}\right)}{\left\|\mathbf{w}_{\min }\right\|^{2}} \leq\left(\ln \left(x_{i}^{(\kappa)}\right)-1\right)\left\|\mathbf{w}_{\min }\right\|^{2}+\frac{\varphi_{i}\left(\mathbf{w}, \mathbf{R}_{i}\right)}{x_{i}^{(\kappa)}}$

Constraint (40c) is thus innerly approximated by

$$
\begin{gathered}
\delta\left[\frac{M-1}{2}\left(\ln \left(x_{i}^{(\kappa)}\right)-1\right)+\delta_{M}\right]\left\|\mathbf{w}_{\min }\right\|^{2} \leq \\
\left(1-\delta(M-1) / 2 x_{i}^{(\kappa)}\right) \varphi_{i}\left(\mathbf{w}, \mathbf{R}_{i}\right) \\
i=1, \ldots, M .
\end{gathered}
$$

Set

$$
i_{\min }=\arg \min _{i=1, \ldots, M}\left\|w_{i}^{(\kappa)}\right\|^{2}
$$

i.e.

$$
\left\|w_{i_{\min }}^{(\kappa)}\right\|^{2}=\min _{i=1, \ldots, M}\left\|w_{i}^{(\kappa)}\right\|^{2}
$$

Verifying numerically that $\frac{M-1}{2}\left(\ln \left(x_{i}^{(\kappa)}\right)-1\right)+\delta_{M} \geq 0$ and $1-\delta(M-1) / 2 x_{i}^{(\kappa)} \geq 0$, we use

$$
\begin{aligned}
\varphi_{i}\left(\mathbf{w}, \mathbf{R}_{i}\right) & \geq \varphi_{i}^{(\kappa)}\left(\mathbf{w}, \mathbf{R}_{i}\right) \\
& \triangleq \ell_{i}^{(\kappa)}\left(\mathbf{w}_{i}, \mathbf{R}_{i}\right)-(1-\delta)^{-1} \sum_{j \neq i}^{M}\left|\bar{h}_{j i}^{H} \mathbf{w}_{j}\right|^{2}-\sigma_{i}^{2}
\end{aligned}
$$

in providing the following convex inner approximation of (42) for each $i=1, \ldots, M$ :

$$
\begin{gathered}
\delta\left[\frac{M-1}{2}\left(\ln \left(x_{i}^{(\kappa)}\right)-1\right)+\delta_{M}\right]\left\|\mathbf{w}_{i_{\min }}\right\|^{2} \leq \\
\left(1-\delta(M-1) / 2 x_{i}^{(\kappa)}\right) \varphi_{i}^{(\kappa)}\left(\mathbf{w}, \mathbf{R}_{i}\right), \\
i=1, \ldots, M .
\end{gathered}
$$

Accordingly, the next feasible point $\left(w^{(\kappa+1)}, R_{l}^{(\kappa+1)}, r_{u}^{(\kappa+1)}\right)$ is generated at the $\kappa$-th iteration by the optimal solution of the convex optimization problem

$$
\begin{gathered}
\max _{\boldsymbol{w}, \mathbf{R}, \mathbf{r}} \min _{i=1, \ldots, M}\left[A_{i}^{(\kappa)}\left(\mathbf{R}_{i}\right)-a_{i}^{(\kappa)}\left(\mathbf{r}_{i}\right)\right] \\
\text { s.t } \quad(3 b),(21 c),(23),(30 b),(41),(44) .
\end{gathered}
$$

The computational complexity of (45) is (14) for $n=M N_{t}+$ $2 M$ and $m=7 M$.

At the same $\kappa$-th iteration, $r_{i}^{(\kappa+1)}$ is found from solving (31) by bisection on $\left[0, r_{u, i}^{(\kappa+1)}\right]$ such that $(32)$, while $R_{i}^{(\kappa+1)}$ is found from solving

$$
\zeta_{i}\left(\mathbf{R}_{i}\right)=0
$$

by bisection on a segment

$$
\left[R_{l, i}, R_{u, i}\right]
$$

such that

$$
-\epsilon_{b} \leq \zeta_{i}\left(R_{i}^{(\kappa+1)}\right) \leq 0
$$

for

$$
\begin{aligned}
\zeta_{i}\left(\mathbf{R}_{i}\right) \triangleq & -\varphi_{i}\left(w^{(\kappa+1)}, \mathbf{R}_{i}\right)+\delta \frac{M-1}{2}\left\|w_{\min }^{(\kappa+1)}\right\|^{2} \\
& \times \ln \frac{\varphi_{i}\left(w^{(\kappa+1)}, \mathbf{R}_{i}\right)}{\left\|w_{\min }^{(\kappa+1)}\right\|^{2}}+\delta \delta_{M}\left\|w_{\min }^{(\kappa+1)}\right\|^{2} .
\end{aligned}
$$

Both $R_{l, i}$ and $R_{u, i}$ in (47) can be easily determined as follow. If $\zeta_{i}\left(R_{l}^{(\kappa+1)}\right)>0$ set $R_{u, i}=R_{l}^{(\kappa+1)}$ and $R_{l, i}=R_{l}^{(\kappa+1)} / \nu$ with the smallest integer $\nu$ such that $\zeta_{i}\left(R_{l}^{(\kappa+1)} / \nu\right)<0$. Otherwise, $\zeta_{i}\left(R_{l}^{(\kappa+1)}\right)<0$ set $R_{l, i}=R_{l}^{(\kappa+1)}$ and $R_{u}^{(\kappa+1)}=$ $\nu R_{l}^{(\kappa+1)}$ with the smallest integer $\nu$ such that $\zeta_{i}\left(\nu R_{l}^{(\kappa+1)}\right)>$ 0 .

\section{Algorithm 4 Path-following algorithm for maximin secrecy} throughput optimization

Initialization: Set $\kappa=0$. Choose an initial feasible point $\left(w^{(0)}, R^{(0)}, r^{(0)}\right)$ for (40) and calculate $R_{\min }^{(0)}$ as the value of the objective function in (40) at $\left(w^{(0)}, R^{(0)}, r^{(0)}\right)$.

repeat

- Solve the convex optimization problem (45) to obtain the solution $\left(w^{(\kappa+1)}, R_{l}^{(\kappa+1)}, r_{u}^{(\kappa+1)}\right)$.

- Solve the nonlinear equations (31) to obtain the roots $r_{i}^{(\kappa)}$.

- Solve the nonlinear equations (46) for $\zeta_{i}\left(\mathbf{R}_{i}\right)$ defined by (49) to obtain the roots $R_{i}^{(\kappa+1)}$.

- Calculate $R_{\min }^{(\kappa+1)}$ as the value of the objective function in (40) at $\left(w^{(\kappa)}, R^{(\kappa+1)}, r^{(\kappa+1)}\right)$.

- Reset $\kappa+1 \rightarrow \kappa$.

until $\frac{\left.R_{\min }^{(\kappa+1)}-R_{\min }^{(\kappa+1)}\right)}{R_{\min }^{(\kappa)}} \leq \epsilon_{\mathrm{tol}}$.

An initial feasible $\left(w^{(0)}, R^{(0)}, r^{(0)}\right)$ can be easily found as follows: taking $w^{(0)}$ and $r^{(0)}$ as the optimal solution of (21) and $R_{i}^{(0)}$ is found from solving $-\epsilon_{b} \leq \zeta_{i}\left(\mathbf{R}_{i}\right) \leq 0$ for

$$
\begin{aligned}
\zeta_{i}\left(\mathbf{R}_{i}\right) \triangleq & -\varphi_{i}\left(w^{(0)}, \mathbf{R}_{i}\right)+\delta \frac{M-1}{2}\left\|w_{\min }^{(0)}\right\|^{2} \\
& \times \ln \frac{\varphi_{i}\left(w^{(0)}, \mathbf{R}_{i}\right)}{\left\|w_{\text {min }}^{(0)}\right\|^{2}}+\delta \delta_{M}\left\|w_{\min }^{(0)}\right\|^{2}
\end{aligned}
$$

by bisection on $\left[R_{l, i}, R_{u, i}\right]$. Here

$$
R_{u, i}=\frac{\left|h_{i e}^{H} w_{i}^{(0)}\right|^{2}}{\sum_{j \neq i}\left|h_{j e}^{H} w_{j}^{(0)}\right|^{2}+\sigma_{e}^{2}}
$$

while $R_{l, i}=R_{u, i} / \nu$ with the smallest integer such that $\zeta_{i}\left(R_{u, i} / n\right)<0$.

Next, we address the EE maximization (4) by the following iterations with the convergence guaranteed.

- Initialization. Use Algorithm 4 to obtain a feasible point $\left(w^{(0)}, R^{(0)}, r^{(0)}\right)$ and define

$$
\theta^{(0)} \triangleq \sum_{i=1}^{M}\left[\ln \left(1+R_{i}^{(0)}\right)-\ln \left(1+r_{i}^{(0)}\right)\right] / \pi\left(w^{(0)}\right) .
$$

- $\kappa$-th iteration. Let $\left(w^{(\kappa)}, R^{(\kappa)}, r^{(\kappa)}\right)$ be a feasible point found from the $(\kappa-1)$ th iteration. Define

$$
\theta^{(\kappa)} \triangleq \sum_{i=1}^{M}\left[\ln \left(1+R_{i}^{(\kappa)}\right)-\ln \left(1+r_{i}^{(\kappa)}\right)\right] / \pi\left(w^{(\kappa)}\right)
$$

and then solve the following convex optimization problem to generate the next feasible point 


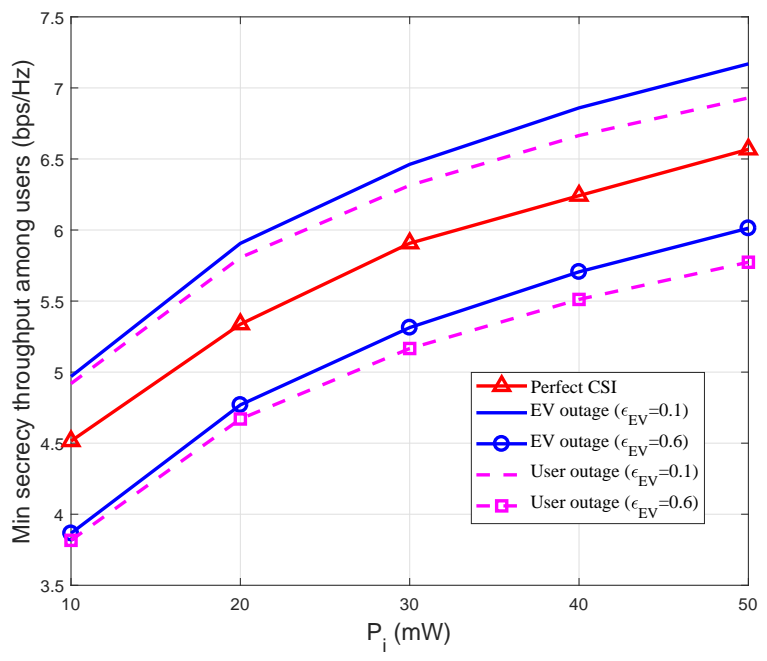

Fig. 1. Minimal secrecy throughput among users versus the transmit power limitation $P_{i}$ with $M=2$.

$$
\begin{aligned}
\left(w^{(\kappa+1)},\right. & \left.R_{l}^{(\kappa+1)}, r_{u}^{(\kappa+1)}\right): \\
\max _{\boldsymbol{w}, \mathbf{r}} & \sum_{i=1}^{M}\left[A_{i}^{(\kappa)}\left(\mathbf{R}_{i}\right)-a_{i}^{(\kappa)}\left(\mathbf{r}_{i}\right)\right]-\theta^{(\kappa)} \pi(\mathbf{w}) \\
& \text { s.t. } \quad(3 b),(21 c),(23),(30 b),(41),(44), \\
& A_{i}^{(\kappa)}\left(\mathbf{R}_{i}\right)-a_{i}^{(\kappa)}\left(\mathbf{r}_{i}\right) \geq c_{i}, i=1, \ldots, M,
\end{aligned}
$$

Further, $r_{i}^{(\kappa+1)}$ is found from solving (31), while $R_{i}^{(\kappa+1)}$ is found from solving (47) till satisfactory of (48) for $\zeta\left(\mathbf{R}_{i}\right)$ defined by (49).

\section{Simulation}

This section presents numerical results to demonstrate the efficiency of the proposed algorithms. Each transmitter is equipped with $N_{t}=4$ antennas. Scenarios of $M \in\{2,5,6\}$ pairs with the noise variance $\sigma_{i}^{2}=\sigma_{e}^{2}=1 \mathrm{~mW}$ are simulated [40], [41]. All entries of channels $h_{j e}$ and $h_{i e}$ in (1) and (2) are generated by independent and identically distributed complex normal random variables of zero mean and unit variance. The drain efficiency $1 / \zeta$ of power amplifier in (4) is $40 \%$ with the circuit power of each transmit antenna $P_{a}=1.25 \mathrm{~mW}$ [30]. The computation tolerance for terminating all proposed Algorithms is $\epsilon_{\text {tol }}=10^{-4}$. The obtained information throughput results are divided by $\ln (2)$ for expressing secrecy throughputs in $\mathrm{bps} / \mathrm{Hz}$ and secure energy efficiencies in bits/J/Hz.

In the below discussion, the terms "Perfect CSI", "EV outage", and "User outage" correspond to the scenarios discussed in Sections III, Section IV with the EV outage probability $\epsilon_{E V} \in\{0.1,0.6\}$ in (18), and section V with the channel error bound $\delta=0.001$ in (35) and user outage probability $\epsilon=0.1$ in (36), respectively.

\section{A. Maximin secrecy throughput optimization}

This subsection analyzes the impact of channel uncertainties to the users' achievable secrecy throughput. Figs. 1, 2 and 3 plot the users' minimum secrecy throughput versus the

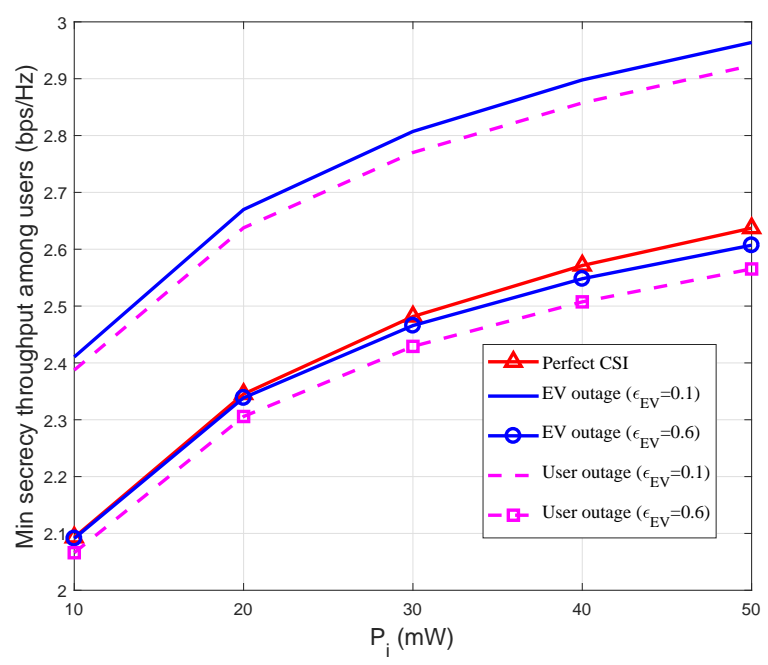

Fig. 2. Minimal secrecy throughput among users versus the transmit power limitation $P_{i}$ with $M=5$.

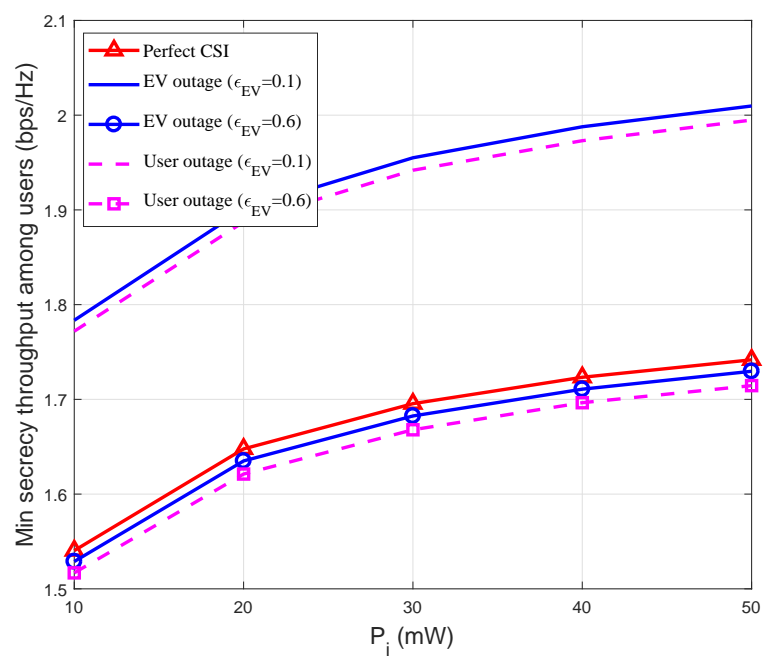

Fig. 3. Minimal secrecy throughput among users versus the transmit power limitation $P_{i}$ with $M=6$.

transmit power limitation $P_{i}$ varying from $10 \mathrm{~mW}$ to $50 \mathrm{~mW}$ for $M=2, M=5$ and $M=6$, respectively. Intuitively, the secrecy throughput increases in the transmitted power limitation $P_{i}$. In each case of $M$, both "EV outage" and "User outage" with the small outage probability $\epsilon_{E V}=0.1$ achieve better secrecy throughputs than "Perfect CSI", but the latter achieves better secrecy throughputs than the formers with the large outage probability $\epsilon_{E V}=0.6$. This outcome is not surprised because the instantaneous wiretapped throughput defined by (6) is actually higher than the throughput outage defined by (18) at small outage probabilities $\epsilon_{E V}$. These figures also show that the secrecy output performance is deteriorated with the increased number of transmitter-user pairs, which leads to a stronger inter-user interference hurting the users' throughput.

Table I provides the average number of iterations required to solve the problem of maximin secrecy throughput optimization 
TABLE I

AVERAGE NUMBER OF ITERATIONS FOR MAXIMIN SECRECY THROUGHPUT OPTIMIZATION WITH $M \in\{2,5,6\}$.

\begin{tabular}{|c|c|c|c|c|c|}
\hline$P_{i}(\mathrm{~mW})$ & 10 & 20 & 30 & 40 & 50 \\
\hline Perfect CSI & $9 / 12 / 13$ & $8 / 15 / 17$ & $10 / 16 / 16$ & $9 / 18 / 19$ & $8 / 18 / 20$ \\
\hline EV outage $\left(\epsilon_{E V}=0.1\right)$ & $5 / 12 / 14$ & $7 / 15 / 17$ & $6 / 17 / 17$ & $7 / 18 / 18$ & $6 / 17 / 20$ \\
\hline User outage $\left(\epsilon_{E V}=0.1\right)$ & $5 / 8 / 8$ & $3 / 9 / 10$ & $4 / 7 / 12$ & $5 / 11 / 12$ & $4 / 10 / 11$ \\
\hline EV outage $\left(\epsilon_{E V}=0.6\right)$ & $8 / 14 / 14$ & $8 / 17 / 18$ & $7 / 17 / 20$ & $8 / 19 / 20$ & $6 / 20 / 22$ \\
\hline User outage $\left(\epsilon_{E V}=0.6\right)$ & $6 / 9 / 7$ & $6 / 12 / 12$ & $3 / 11 / 10$ & $4 / 12 / 15$ & $4 / 13 / 14$ \\
\hline
\end{tabular}

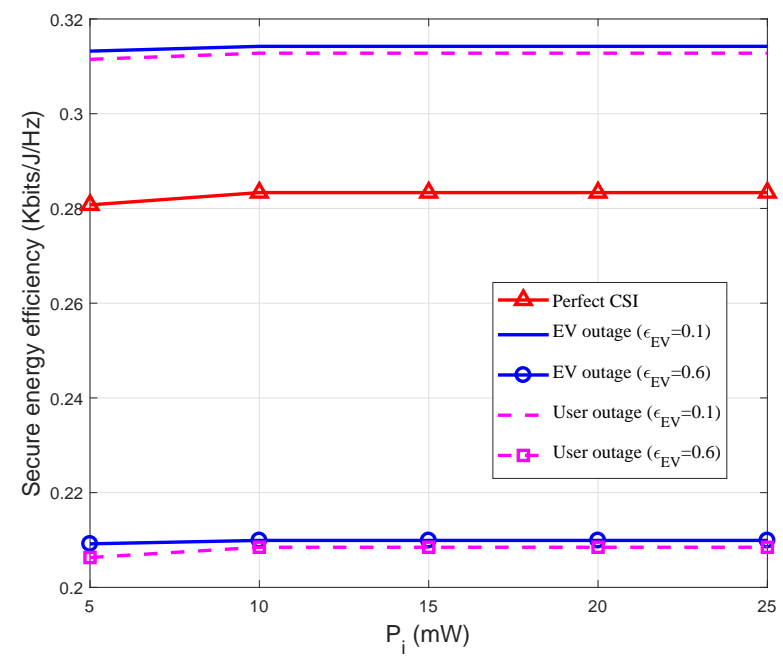

Fig. 4. Energy efficiency versus the transmit power limitation $P_{i}$ with $M=$

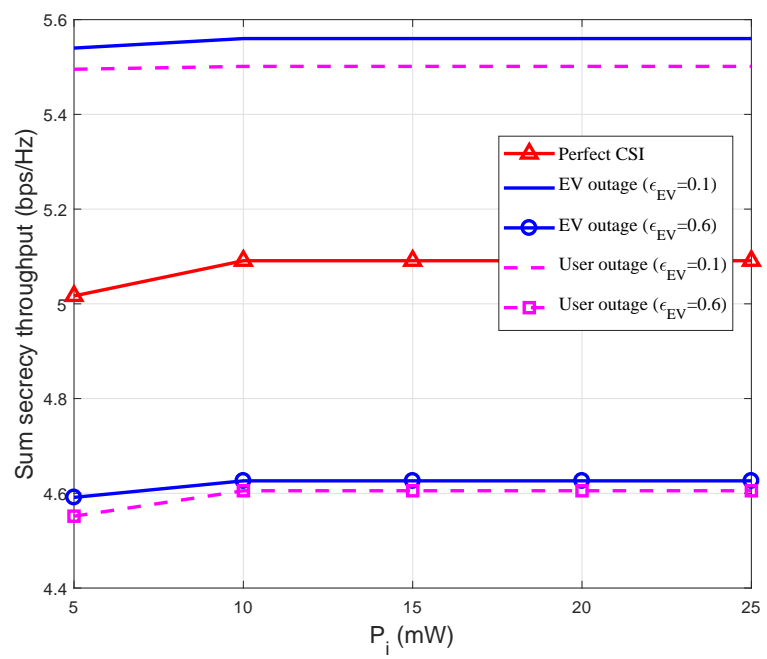

Fig. 5. Sum throughput versus the transmit power limitation $P_{i}$ with $M=2$.

for the above three cases with $M=2, M=5$ and $M=6$, respectively. On average, the proposed algorithms converge in less than 10, 20 and 22 iterations, for $M=2, M=5$ and $M=6$, respectively.

\section{B. Secure energy efficiency maximization}

This subsection examines the performance of the proposed SEE maximization algorithms. The threshold $c_{i}$ in $(4 \mathrm{~b})$ for QoS is $2 \mathrm{bps} / \mathrm{Hz}, 1 \mathrm{bps} / \mathrm{Hz}$ and $0.6 \mathrm{bps} / \mathrm{Hz}$ for $M=2$,

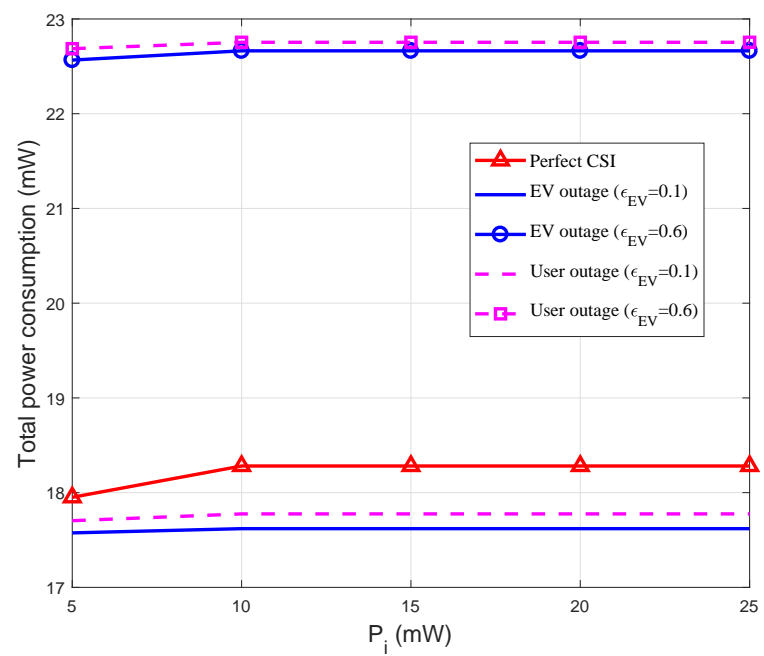

Fig. 6. Total power consumption versus the transmit power limitation $P_{i}$ with $M=2$.

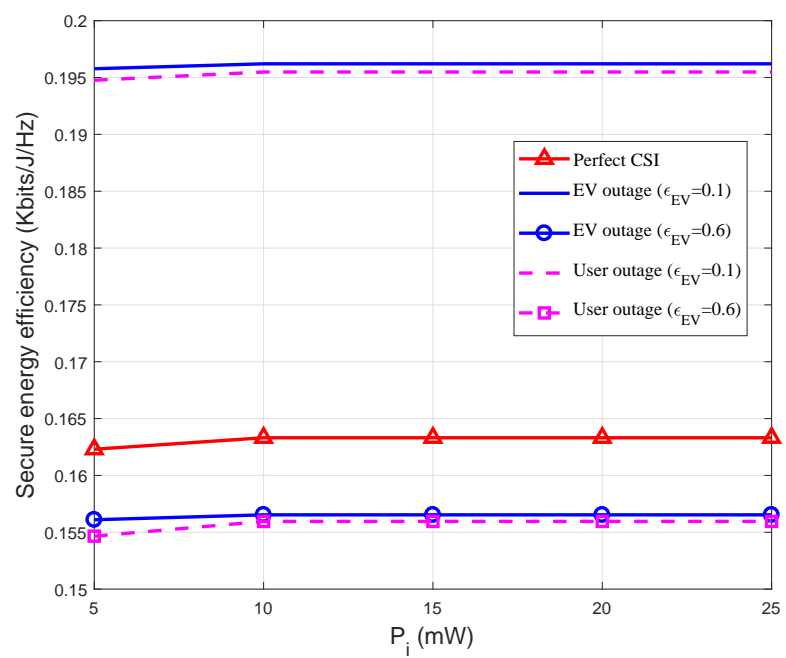

Fig. 7. Energy efficiency versus the transmit power limitation $P_{i}$ with $M=$ 5 .

$M=5$ and $M=6$, respectively. The transmit power limitation $P_{i}$ varies from $5 \mathrm{~mW}$ to $25 \mathrm{~mW}$. Fig. 4 shows that "EV outage" with the small outage probability $\epsilon_{E V}=$ 0.1 significantly outperforms other cases. The corresponding sum secrecy throughput and total transmit power plotted in Figs. 5 and 6 particularly explain this. "EV outage" with $\epsilon_{E V}=0.1$ achieves higher sum secrecy throughput in Fig. 5 and consumes less power in Fig. 6. Furthermore, the SEE 


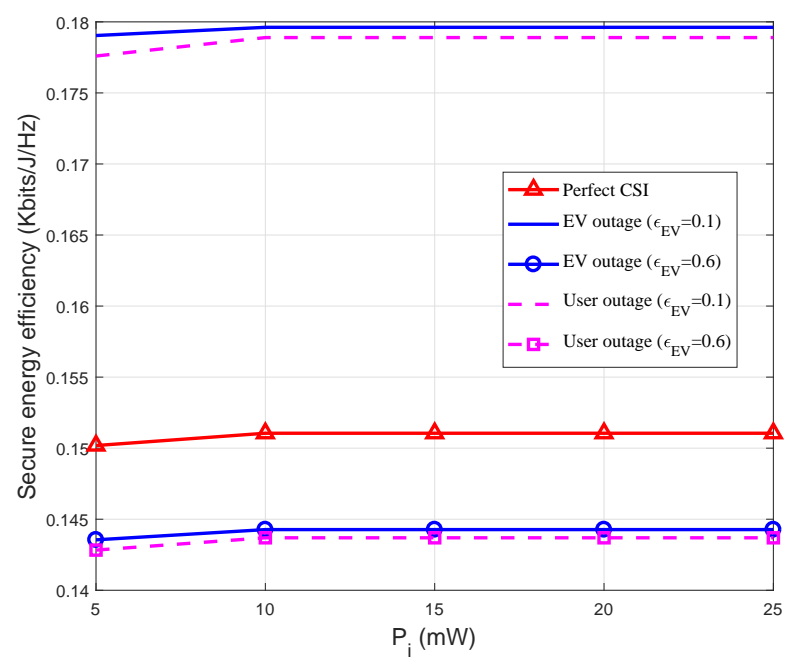

Fig. 8. Energy efficiency versus the transmit power limitation $P_{i}$ with $M=$ 6.

performances saturates when the transmit power limitation exceeds the threshold $10 \mathrm{~mW}$. In the region of small transmit power limitation, the denominator of SEE is dominated by the circuit power so the SEE is maximized by maximizing the sum secrecy throughput in the numerator. However, in larger regions of transmit power limitation, the denominator of SEE becomes to be dominated by the actual transmit power, which by Fig. 6 saturates after $P_{i}=10 \mathrm{~mW}$, making the sum secrecy throughput and SEE behave similarly in Figs. 4 and 5. Further, by Fig. 7 and 8, SEE follows a similar pattern for $M=5$ and $M=6$, respectively.

Lastly, the average number of iterations is provided by Table II, which particularly shows that our proposed SEE maximization algorithm on average converges in less than 16, 24 and 28 iterations for $M=2, M=5$ and $M=6$, respectively.

\section{CONCLUSIONS}

For a wireless network of multiple transmitter-user pairs overhear by an eavesdropper, we have considered the beamforming design to maximize either the users' secrecy throughput or the network's secure energy efficiency under QoS constraints in terms of users' secrecy throughput thresholds. At different levels of channel knowledge, we have developed path-following algorithms of low complexity but rapid convergence for computation. The provided simulations have not only shown the efficiency of the developed algorithms but also linked the outage probability with the secrecy degree. Extensions to multi-cell coordinated beamforming are underway.

\section{APPENDIX I: OUTAGE PROBABILITY INEQUALITIES}

We derive bounds for

$$
\begin{aligned}
& \operatorname{Prob}\left(\frac{a}{\delta \sum_{i=1}^{M}\left|\left\langle\chi_{i}, \mathbf{w}_{i}\right\rangle\right|^{2}+b}<r\right) \\
\Leftrightarrow & \operatorname{Prob}\left(a / r-b<\delta \sum_{i=1}^{M}\left|\left\langle\chi_{i}, \mathbf{w}_{i}\right\rangle\right|^{2}\right)
\end{aligned}
$$

for $a>0, b>0$ and $r>0$. Here $\chi_{i} \in \mathcal{C N}(0, I)$ while $\mathbf{w}_{i}$ are deterministic complex vectors.

Note that

$$
\left|\left\langle\chi_{i}, \mathbf{w}_{i}\right\rangle\right|^{2}=\left\|\mathbf{w}_{i}\right\|^{2}\left|\left\langle\chi_{i}, \mathbf{w}_{i} /|| \mathbf{w}_{i}||\right\rangle\right|^{2}=\left\|\mathbf{w}_{i}\right\|^{2} p_{i}
$$

where $p_{i}$ is an exponential distribution with the unit mean.

As

$$
\frac{a}{\delta \sum_{i=1}^{M}\left|\left\langle\chi_{i}, \mathbf{w}_{i}\right\rangle\right|^{2}+b}<\frac{a}{b}
$$

the probability in (51) is not zero if and only if

$$
r<a / b \Leftrightarrow a / r-b>0 .
$$

For

$$
\left\|\mathbf{w}_{\min }\right\|^{2} \triangleq \min _{i=1, \ldots, M}\left\|\mathbf{w}_{i}\right\|^{2} .
$$

it follows that

$$
\begin{aligned}
& \operatorname{Prob}\left(\sum_{i=1}^{M}\left|\left\langle\chi_{i}, \mathbf{w}_{i}\right\rangle\right|^{2}<(a / r-b) / \delta\right)= \\
& \operatorname{Prob}\left(\sum_{i=1}^{M}\left\|\mathbf{w}_{i}\right\|^{2} p_{i}<(a / r-b) / \delta\right) \leq \\
& \operatorname{Prob}\left(\sum_{i=1}^{M}\left\|\mathbf{w}_{\min }\right\|^{2} p_{i}<(a / r-b) / \delta\right)= \\
& \operatorname{Prob}\left(\sum_{i=1}^{M} p_{i}<(a / r-b) / \delta\left\|\mathbf{w}_{\min }\right\|^{2}\right)= \\
& \int_{\sum_{i=1}^{M} t_{i}<\frac{a-r b}{\delta\left\|\mathbf{w}_{\min }\right\|^{2}}} \prod_{i=1}^{M} e^{-t_{i}} d t_{1} \cdots d t_{M} .
\end{aligned}
$$

Using the representation

$$
u(x)=\frac{1}{2 \pi} \int_{-\infty}^{\infty} \frac{e^{x(\jmath \omega+\beta)}}{\jmath \omega+\beta} d \omega
$$

for the unit step function [42] leads to

$$
\begin{array}{r}
\int_{i=1}^{M} t_{i}<\frac{a-r b}{\delta\left\|\mathbf{w}_{\min }\right\|^{2}} \prod_{i=1}^{M} e^{-t_{i}} d t_{1} \cdots d t_{M}= \\
\frac{1}{2 \pi} \int_{0}^{\infty} \cdots \int_{0}^{\infty} \int_{-\infty}^{\infty} \frac{e^{\left[\frac{a / r-b}{\delta \|\left.\mathbf{w}_{\min }\right|^{2}}-\sum_{i=1}^{M} t_{i}\right](\jmath \omega+\beta)}}{\jmath \omega+\beta}
\end{array}
$$


TABLE II

AVERAGE NUMBER OF ITERATIONS FOR SECURE ENERGY EFFICIENCY MAXIMIZATION WITH $M \in\{2,5,6\}$.

\begin{tabular}{|c|c|c|c|c|c|}
\hline$P_{i}(\mathrm{~mW})$ & 5 & 10 & 15 & 20 & 25 \\
\hline Perfect CSI & $10 / 14 / 19$ & $13 / 18 / 23$ & $14 / 20 / 25$ & $14 / 21 / 27$ & $15 / 22 / 28$ \\
\hline EV outage $\left(\epsilon_{E V}=0.1\right)$ & $10 / 14 / 16$ & $12 / 17 / 21$ & $12 / 18 / 21$ & $13 / 19 / 22$ & $14 / 19 / 22$ \\
\hline User outage $\left(\epsilon_{E V}=0.1\right)$ & $3 / 7 / 9$ & $4 / 7 / 9$ & $5 / 9 / 10$ & $3 / 8 / 9$ & $6 / 10 / 11$ \\
\hline EV outage $\left(\epsilon_{E V}=0.6\right)$ & $11 / 16 / 18$ & $13 / 19 / 23$ & $15 / 22 / 25$ & $16 / 24 / 26$ & $16 / 23 / 28$ \\
\hline User outage $\left(\epsilon_{E V}=0.6\right)$ & $5 / 8 / 11$ & $7 / 8 / 11$ & $4 / 9 / 10$ & $6 / 10 / 12$ & $5 / 10 / 12$ \\
\hline
\end{tabular}

$$
\begin{aligned}
& \times\left(\prod_{i=1}^{M} e^{-t_{i}} d t_{i}\right) d \omega= \\
& \frac{1}{2 \pi} \int_{0}^{\infty} \cdots \int_{0}^{\infty} \int_{-\infty}^{\infty} \prod_{i=1}^{M} e^{-t_{i}(1+\jmath \omega+\beta)} d t_{1} \ldots d t_{M} \\
& \times \frac{e^{(\jmath \omega+\beta) \frac{a / r-b}{\delta\left\|\mathbf{w}_{\min }\right\|^{2}}}}{\jmath \omega+\beta} d \omega= \\
& \frac{1}{2 \pi} \int_{-\infty}^{\infty} \frac{1}{(1+\jmath \omega+\beta)^{M}} \frac{e^{(\jmath \omega+\beta) \frac{a / r-b}{\delta\left\|\mathbf{w}_{\min }\right\|^{2}}}}{\jmath \omega+\beta} d \omega= \\
& \frac{1}{2 \pi} \int_{-\infty}^{\infty}\left[\frac{1}{\jmath \omega+\beta}-\sum_{i=1}^{M} \frac{1}{(1+\jmath \omega+\beta)^{i}}\right] \\
& \times e^{(\jmath \omega+\beta) \frac{a / r-b}{\delta\left\|\mathbf{w}_{\min }\right\|^{2}}} d \omega, \\
& \operatorname{Prob}\left(\sum_{i=1}^{M}\left|\left\langle\chi_{i}, \mathbf{w}_{i}\right\rangle\right|^{2}<(a / r-b) / \delta\right) \leq \\
& 1-e^{-(a / r-b) / \delta\left\|\mathbf{w}_{\min }\right\|^{2}} \sum_{i=1}^{M} \frac{(a / r-b)^{i-1}}{\Gamma(i)\left(\delta\left\|\mathbf{w}_{\min }\right\|^{2}\right)^{i-1}}, \\
& \operatorname{Prob}\left(\sum_{i=1}^{M}\left|\left\langle\chi_{i}, \mathbf{w}_{i}\right\rangle\right|^{2} \geq(a / r-b) / \delta\right)= \\
& 1-\operatorname{Prob}\left(\sum_{i=1}^{M}\left|\left\langle\chi_{i}, \mathbf{w}_{i}\right\rangle\right|^{2}<(a / r-b) / \delta\right) \geq \\
& 1-\left[1-e^{-(a / r-b) / \delta\left\|\mathbf{w}_{\min }\right\|^{2}} \sum_{i=1}^{M} \frac{(a / r-b)^{i-1}}{\Gamma(i)\left(\delta\left\|\mathbf{w}_{\min }\right\|^{2}\right)^{i-1}}\right]= \\
& e^{-(a / r-b) / \delta\left\|\mathbf{w}_{\min }\right\|^{2}} \sum_{i=1}^{M} \frac{(a / r-b)^{i-1}}{\Gamma(i)\left(\delta\left\|\mathbf{w}_{\min }\right\|^{2}\right)^{i-1}} .
\end{aligned}
$$

$$
\frac{1}{x(1+x)^{M}}=\frac{1}{x}-\sum_{i=1}^{M} \frac{1}{(1+x)^{i}},
$$

which can be proved by mathematical induction. Indeed, it is obvious that

$$
\frac{1}{x(1+x)}=\frac{1}{x}-\frac{1}{1+x},
$$

i.e. (55) holds true for $M=1$. Suppose that (55) is true for $M=n$, i.e.

$$
\frac{1}{x(1+x)^{n}}=\frac{1}{x}-\sum_{i=1}^{n} \frac{1}{(1+x)^{i}}
$$

Then

$$
\begin{aligned}
\frac{1}{x}-\sum_{i=1}^{n+1} \frac{1}{(1+x)^{i}} & =\left(\frac{1}{x}-\sum_{i=1}^{n} \frac{1}{(1+x)^{i}}\right)-\frac{1}{(1+x)^{n+1}} \\
& =\frac{1}{x(1+x)^{n}}-\frac{1}{(1+x)^{n+1}} \\
& =\frac{1}{x(1+x)^{n+1}},
\end{aligned}
$$

i.e. (55) is true for $M=n+1$, completing the proof for (55).

Furthermore, by [42, (28)-(29)]

$$
\begin{gathered}
\frac{1}{2 \pi} \int_{-\infty}^{\infty} \frac{e^{x(\jmath \omega+\beta)}}{\jmath \omega+\beta} d \omega=1 \quad \text { for } \quad x>0 \\
\frac{1}{2 \pi} \int_{-\infty}^{\infty} \frac{e^{x(\jmath \omega+\beta)}}{(1+\jmath \omega+\beta)^{i}} d \omega=\frac{e^{-x} x^{i-1}}{\Gamma(i)} \quad \text { for } \quad x>0
\end{gathered}
$$

Analogously,

$$
\begin{array}{r}
\operatorname{Prob}\left(\sum_{i=1}^{M}\left|\left\langle\chi_{i}, \mathbf{w}_{i}\right\rangle\right|^{2}<(a / r-b) / \delta\right) \geq \\
1-e^{-(a / r-b) / \delta\left\|\mathbf{w}_{\max }\right\|^{2}} \sum_{i=1}^{M} \frac{(a / r-b)^{i-1}}{\Gamma(i)\left(\delta\left\|\mathbf{w}_{\max }\right\|^{2}\right)^{i-1}},
\end{array}
$$

for

$$
\left\|\mathbf{w}_{\max }\right\|^{2} \triangleq \max _{i=1, \ldots, M}\left\|\mathbf{w}_{i}\right\|^{2}
$$

Therefore, we arrive at the following result.

Theorem 1: The following two-sided inequalities hold true:

$$
\begin{aligned}
& e^{-(a / r-b) / \delta\left\|\mathbf{w}_{\min }\right\|^{2}} \sum_{i=1}^{M} \frac{(a / r-b)^{i-1}}{\Gamma(i)\left(\delta\left\|\mathbf{w}_{\min }\right\|^{2}\right)^{i-1}} \leq(5 \\
& \operatorname{Prob}\left(a / r-b<\delta \sum_{i=1}^{M}\left|\left\langle\chi_{i}, \mathbf{w}_{i}\right\rangle\right|^{2}\right)= \\
& \operatorname{Prob}\left(\frac{a}{\delta \sum_{i=1}^{M}\left|\left\langle\chi_{i}, \mathbf{w}_{i}\right\rangle\right|^{2}+b} \leq r\right) \leq \\
& e^{-(a / r-b) / \delta\left\|\mathbf{w}_{\max }\right\|^{2}} \sum_{i=1}^{M} \frac{(a / r-b))^{i-1}}{\Gamma(i)\left(\delta\left\|\mathbf{w}_{\max }\right\|^{2}\right)^{i-1}} .
\end{aligned}
$$


Now, by Cauchy inequality

$$
\begin{aligned}
& \sum_{i=1}^{M} \frac{(a / r-b)^{i-1}}{\Gamma(i)\left(\delta\left\|\mathbf{w}_{\min }\right\|^{2}\right)^{i-1}} \geq \\
& M\left(\prod_{i=1}^{M} \frac{(a / r-b)^{i-1}}{\Gamma(i)\left(\delta\left\|\mathbf{w}_{\min }\right\|^{2}\right)^{i-1}}\right)^{1 / M}= \\
& \frac{M}{\left(\prod_{i=1}^{M} \Gamma(i)\right)^{1 / M}}\left(\frac{(a / r-b)}{\delta\left\|\mathbf{w}_{\min }\right\|^{2}}\right)^{(M-1) / 2} .
\end{aligned}
$$

Therefore, it follows from (59) that

$$
\begin{aligned}
& e^{-(a / r-b) / \delta\left\|\mathbf{w}_{\min }\right\|^{2}} \frac{M}{\left(\prod_{i=1}^{M} \Gamma(i)\right)^{1 / M}} \\
& \times\left(\frac{(a / r-b)}{\delta\left\|\mathbf{w}_{\min }\right\|^{2}}\right)^{(M-1) / 2} \leq \\
& \operatorname{Prob}\left(\frac{a}{\delta \sum_{i=1}^{M}\left|\left\langle\chi_{i}, \mathbf{w}_{i}\right\rangle\right|^{2}+b} \leq r\right) \text {. }
\end{aligned}
$$

Then

$$
\begin{aligned}
& \max \left\{r: \operatorname{Prob}\left(\frac{a}{\delta \sum_{i=1}^{M}\left|\left\langle\chi_{i}, \mathbf{w}_{i}\right\rangle\right|^{2}+b} \leq r\right)\right. \\
& \leq \epsilon\} \geq \\
& \max \left\{r: e^{-(a / r-b) / \delta\left\|\mathbf{w}_{\min }\right\|^{2}} \frac{M}{\left(\prod_{i=1}^{M} \Gamma(i)\right)^{1 / M}}\right. \\
& \left.\times\left(\frac{(a / r-b)}{\delta\left\|\mathbf{w}_{\min }\right\|^{2}}\right)^{(M-1) / 2} \leq \epsilon\right\}=(62) \\
& \max \left\{r:-\frac{a / r-b}{\delta\left\|\mathbf{w}_{\min }\right\|^{2}}\right. \\
& +\left(\ln M-\frac{1}{M} \sum_{i=1}^{M} \ln \Gamma(i)\right) \\
& +\frac{M-1}{2}\left(\ln (a / r-b)-\ln \delta-\ln \left\|\mathbf{w}_{\min }\right\|^{2}\right) \\
& \leq \ln \epsilon\}=(63) \\
& \max \left\{r:-\frac{a / r-b}{\left\|\mathbf{w}_{\min }\right\|^{2}}+\delta(\ln M\right. \\
& \left.-\frac{1}{M} \sum_{i=1}^{M} \ln \Gamma(i)\right)+\delta \frac{M-1}{2}(\ln (a / r-b) \\
& \left.\left.-\ln \delta-\ln \left\|\mathbf{w}_{\min }\right\|^{2}\right) \leq \delta \ln \epsilon\right\}= \\
& \max \left\{r:-\frac{a / r-b}{\left\|\mathbf{w}_{\min }\right\|^{2}}+\delta \frac{M-1}{2}\right. \\
& \times\left(\ln (a / r-b)-\ln \left\|\mathbf{w}_{\min }\right\|^{2}\right) \leq \delta(\ln \epsilon-\ln M
\end{aligned}
$$

$$
\left.\left.+\frac{1}{M} \sum_{i=1}^{M} \ln \Gamma(i)+\frac{M-1}{2} \ln \delta\right)\right\} .
$$

Note that for $M=1$ it follows from (59) and (60) that

$$
\operatorname{Prob}\left(\frac{a}{|\langle\chi, \mathbf{w}\rangle|^{2}+b}<r\right)=e^{-(a / r-b) /\|\mathbf{w}\|^{2}},
$$

which is a known result since $|\langle\chi, \mathbf{w}\rangle|^{2}$ is an exponential distribution with mean $\|\mathbf{w}\|^{2}$ :

$$
\begin{aligned}
\operatorname{Prob}\left(\frac{a}{|\langle\chi, \mathbf{w}\rangle|^{2}+b}<r\right) & =\operatorname{Prob}\left(a / r-b<\left.\langle\chi, \mathbf{w}\rangle\right|^{2}\right) \\
& =\int_{a / r-b}^{\infty} e^{-t /\|\mathbf{w}\|^{2}} /\|\mathbf{w}\|^{2} d t \\
& =e^{-(a / r-b) /\|\mathbf{w}\|^{2}} .
\end{aligned}
$$

Particularly,

$$
\begin{gathered}
\max \left\{r: \operatorname{Prob}\left(\frac{a}{\delta|\langle\chi, \mathbf{w}\rangle|^{2}+b}<r\right) \leq\right. \\
\epsilon\}=\frac{a}{b+\delta|| \mathbf{w} \|^{2} \ln \epsilon^{-1}} .
\end{gathered}
$$

\section{APPENDIX II: BERNSTEIN-TYPE INEQUALITY AND ITS CONSERVATIVENESS}

There is an approach, which is based on the following Bernstein-type inequality [20] of rough estimation.

Theorem 2: [20, Lemma 0.2] Suppose that $A$ is a symmetric matrix and $z$ is Gaussian with zero mean and identity covariance. Then

$$
\begin{array}{r}
\operatorname{Prob}\left(z^{H} A z \geq \operatorname{trace}(A)+2\|A\| \sqrt{x}+2 \lambda_{\max }^{+}(A) x\right) \leq \\
\exp (-x),
\end{array}
$$

where $\lambda_{\max }^{+}(A)=\max \left\{\lambda_{\max }, 0\right\}$.

One can use inequality (66) for an inner approximation of the set

$$
R_{\epsilon}(a, b) \triangleq\left\{r: \operatorname{Prob}\left(\delta \sum_{i=1}^{M}\left|\left\langle\chi_{i}, \mathbf{w}_{i}\right\rangle\right|^{2}>\frac{a}{r}-b\right) \leq \epsilon\right\} .
$$

By setting $z=\left[\chi_{i}^{T}\right]_{i=1, \ldots, M}^{T}$ and

$$
A=\operatorname{diag}\left[\mathbf{w}_{i} \mathbf{w}_{i}^{H}\right]_{i=1, \ldots, M}
$$

we have $\sum_{i=1}^{M}\left|\left\langle\chi_{i}, \mathbf{w}_{i}\right\rangle\right|^{2}=z^{H} A z$ and $\operatorname{trace}(A)=$ $\sum_{i=1}^{M}\left\|\mathbf{w}_{i}\right\|^{2}$, and

$$
\lambda_{\max }(A)=\max _{i=1, \ldots, M}\left[\left\|\mathbf{w}_{i}\right\|^{2}\right] \text { and }\|A\|=\sqrt{\sum_{i=1}^{M}\left\|\mathbf{w}_{i}\right\|^{4}} .
$$

According to (66)

$$
\begin{aligned}
\operatorname{Prob}\left(z^{H} A z \geq \operatorname{trace}(A)+2\|A\| \sqrt{\ln \epsilon^{-1}}\right. & \\
+ & \left.2 \lambda_{\max }(A) \ln \epsilon^{-1}\right) \leq \epsilon .
\end{aligned}
$$

Therefore, $r \in R_{\epsilon}(a, b)$ if

$$
\begin{aligned}
\left(\frac{a}{r}-b\right) / \delta & \geq \operatorname{trace}(A)+2\|A\| \sqrt{\ln \epsilon^{-1}} \\
& +2 \lambda_{\max }(A) \ln \epsilon^{-1}
\end{aligned}
$$




$$
\begin{aligned}
\Leftrightarrow \quad\left(\frac{a}{r}-b\right) / \delta & \geq \sum_{i=1}^{M}\left\|\mathbf{w}_{i}\right\|^{2}+2 \sqrt{\sum_{i=1}^{M}\left\|\mathbf{w}_{i}\right\|^{4} \sqrt{\ln \epsilon^{-1}}} \\
& +2 \max _{i=1, \ldots, M}\left[\left\|\mathbf{w}_{i}\right\|^{2}\right] \ln \epsilon^{-1} \\
\Leftrightarrow \quad r \leq a /[b+ & \delta\left(\sum_{i=1}^{M}\left\|\mathbf{w}_{i}\right\|^{2}+2 \sqrt{\sum_{i=1}^{M}\left\|\mathbf{w}_{i}\right\|^{4} \sqrt{\ln \epsilon^{-1}}}\right. \\
+ & \left.\left.\max _{i=1, \ldots, M}\left[\left\|\mathbf{w}_{i}\right\|^{2}\right] \ln \epsilon^{-1}\right)\right]
\end{aligned}
$$

which is too conservative compared with (64). For instance, for $M=1$, (68) means

$$
r=a /\left(b+\delta\|\mathbf{w}\|^{2}\left(1+2 \sqrt{\ln \epsilon^{-1}}+2 \ln \epsilon^{-1}\right)\right)
$$

which is very conservative compared with (65).

\section{APPENDIX III: BASIC DETERMINISTIC INEQUALITIES}

For every $x>0, y>0, \bar{x}>0$ and $\bar{y}>0$,

$$
\begin{array}{r}
\ln (1+1 / x y) \\
\ln (1+1 / \bar{x} \bar{y})+\frac{1 / \bar{x} \bar{y}}{1+1 / \bar{x} \bar{y}}(2-x / \bar{x}-y / \bar{y}),
\end{array}
$$

which follows from the convexity of function $\ln (1+1 / x y)$ in the domain $\{x>0, y>0\}$. Furthermore,

$$
\ln (1+x / y) \leq \ln (1+\bar{x} / \bar{y})+\frac{1}{1+\bar{x} / \bar{y}}(x / y-\bar{x} / \bar{y}),
$$

which follows from the concavity of function $\ln (1+z)$ in the domain $\{z>0\}$. Lastly, based on the inequality

$$
x^{2} / t \geq 2(\bar{x} / \bar{t}) x-\left(\bar{x}^{2} / \bar{t}^{2}\right) t \quad \forall x>0, \bar{x}>0, t>0, \bar{t}>0
$$

that follows from the convexity of $x^{2} / t$, we have the following inequality

$$
\begin{array}{r}
\frac{r}{\|\mathbf{w}\|^{2}} \geq 2\left(\sqrt{\bar{r}} /\|\bar{w}\|^{2}\right) \sqrt{r}-\left(\bar{r} /\|\bar{w}\|^{4}\right)\|\mathbf{w}\|^{2} \\
\forall r>0, \bar{r}>0, \mathbf{w} \in \mathbb{C}^{N}, \bar{w} \in \mathbb{C}^{N} .
\end{array}
$$

\section{REFERENCES}

[1] A. G. Fragkiadakis, E. Z. Tragos, and I. G. Askoxylakis, "A survey on security threats and detection techniques in cognitive radio networks," IEEE Commun. Surveys Tuts., vol. 15, pp. 428-445, Jan 2013.

[2] A. Mukherjee, S. A. A. Fakoorian, J. Huang, and A. L. Swindlehurst, "Principles of physical layer security in multiuser wireless networks: A survey," IEEE Commun. Surveys Tuts., vol. 16, p. 15501573, Feb 2014.

[3] Y. Liang, H. V. Poor, and S. Shamai, "Secure communication over fading channels," IEEE Trans. Inf. Theory, vol. 54, pp. 2470-2492, June 2008.

[4] R. Bassily et al, "Cooperative security at the physical layer: A summary of recent advances," IEEE Signal Process. Mag., vol. 30, pp. 16-28, May 2013.

[5] H. V. Poor, "Information and inference in the wireless physical layer," IEEE Commun. Mag., vol. 19, pp. 40-47, Feb. 2012.

[6] H. V. Poor and R. F. Schaefer, "Wireless physical layer security," Proc. Nat. Acad. Sciences USA, vol. 114, no. 1, pp. 19-26, 2017.

[7] A. Chorti, S. M. Perlaza, Z. Han, and H. V. Poor, "On the resilence of wireless multiuser networks to passive and active eavesdroppers," IEEE J. Sel. Areas. Commun., vol. 31, no. 9, pp. 1850-1863, 2013.
[8] A. Mukherjee and A. L. Swindlehurst, "Robust beamforming for security in mimo wiretap channels with imperfect CSI," IEEE Trans. Signal Process., vol. 59, pp. 351-361, Jan. 2011.

[9] Q. Li and W. K. Ma, "Spatially selective artificial-noise aided transmit optimization for MISO multi-eves secrecy rate maximization," IEEE Trans. Signal Process., vol. 61, pp. 2704-2717, May 2013.

[10] L. Liu, R. Zhang, and K.-C. Chua, "Secrecy wireless information and power transfer with MISO beamforming," IEEE Trans. Signal Process., vol. 62, pp. 1850-1863, Apr. 2014.

[11] P. Zhao, M. Zhang, H. Yu, H. Luo, and W. Chen, "Robust beamforming design for sum secrecy rate optimization in MU-MISO networks," IEEE Trans. Info. Forens. Sec., vol. 10, pp. 1812-1823, Sept. 2015.

[12] Z. Chu, Z. Zhu, M. Johnston, and S. L. Goff, "Simultaneous wireless information power transfer for MISO secrecy channel," IEEE Trans. Veh. Tech., vol. 65, pp. 6913-6925, Sept. 2016.

[13] A. H. Phan, H. D. Tuan, H. H. Kha, and D. T. Ngo, "Nonsmooth optimization for efficient beamforming in cognitive radio multicast transmission," IEEE Trans. Signal Processing, vol. 60, pp. 2941-2951, Jun. 2012.

[14] A. A. Nasir, H. D. Tuan, T. Q. Duong, and H. V. Poor, "Secrecy rate beamforming for multicell networks with information and energy harvesting," IEEE Trans. Signal Process., vol. 65, pp. 677-689, Feb. 2017.

[15] A. A. Nasir, H. D. Tuan, T. Q. Duong, and H. V. Poor, "Secure and energy-efficient beamforming for simultaneous information and energy transfer," IEEE Trans. Wirel. Commun., vol. 16, pp. 7523-7537, Nov. 2017.

[16] J. Park, Y. Sung, D. Kim, and H. V. Poor, "Outage probability and outage-based robust beamforming for MIMO interference channels with imperfect channel state information," IEEE Trans. Wireless Commun., vol. 11, pp. 3561-3573, Oct. 2012.

[17] K. Wang, A. So, T. Chang, W. Ma, and C. Chi, "Outage constrained robust transmit optimization for multiuser MISO downlinks: Tractable approximations by conic optimization," IEEE Trans. Signal Process., vol. 62, pp. 5690-5705, Nov. 2014.

[18] Z. Zhu, Z. Chu, Z. Wang, and I. Lee, "Outage constrained robust beamforming for secure broadcasting systems with energy harvesting," IEEE Trans. Wirel. Commun., vol. 15, pp. 7610-7620, Nov. 2016.

[19] T. A. Le, Q. T. Vien, H. X. Nguyen, D. W. K. Ng, and R. Schober, "Robust chance-constrained optimization for power-efficient and secure swipt systems," IEEE Trans. Green Commun. and Network, vol. 1, pp. 333-346, Jun. 2017.

[20] I. Bechar, "A Bernstein-type inequality for stochastic processes of quadartic forms of Gaussian variable," Preprint, INRIA Sophia Antiplois, 2009.

[21] E. Stathakis, J. Jalden, L. K. Rasmussen, and M. Skoglund, "Outage region characterization for beamforming in MISO interference networks with imperfect CSI," IEEE Signal Process. Lett., vol. 22, pp. 2378-2382, Dec. 2015.

[22] R. L. G. Cavalcante, S. Stanczak, M. Schubert, A. Eisenlatter, and U. Turke, "Toward energy-efficienct $5 \mathrm{G}$ wireless communications technologies," IEEE Signal Process. Mag., vol. 13, pp. 24-34, Nov. 2014.

[23] C. I, C. Rowell, S. Han, Z. Xu, G. Li, and Z. Pan, "Toward green and soft: A 5G perspective," IEEE Commun. Mag., vol. 13, pp. 66-73, Feb. 2014.

[24] D. W. K. Ng, E. S. Lo, and R. Schober, "Energy-efficient resource allocation for secure ofdma systems," IEEE Trans. Vehic. Techn., vol. 61, pp. 2572-2585, Jul. 2012.

[25] D. Wang, B. Bai, W. Chen, and Z. Han, "Achieving high energy efficiency and physical-layer security in AF relaying," IEEE Trans. Wireless Commun., vol. 15, pp. 740-752, Jan 2016.

[26] N. Zhao, F. R. Yu, and H. Sun, "Adaptive energy-efficient power allocation in green interference-alignment-based wireless networks," IEEE Trans. Veh. Technol., vol. 64, pp. 4268-4281, Sept. 2015.

[27] T. T. Vu, H. H. Kha, and H. D. Tuan, "Transceiver design for optimizing the energy efficiency in multiuser MIMO channels," IEEE Commun. Lett., vol. 20, pp. 1507-1510, Aug. 2016.

[28] Q.-D. Vu, L.-N. Tran, R. Farrell, and E.-K. Hong, "Energy-efficient zero-forcing precoding design for small-cell networks," IEEE Trans. Commun., vol. 64, pp. 790-804, Feb 2016.

[29] A. Kalantari, S. Maleki, S. Chatzinotas, and B. Ottersten, "Secrecy energy efficiency optimization for MISO and SISO communication networks," in Proc. IEEE 16th Inter. Workshop on Signal Process. Advances in Wireless Commun.(SPAWC), pp. 21-25, 2015.

[30] A. Zappone, P.-H. Lin, and E. Jorswieck, "Energy efficiency of confidential multi-antenna systems with artificial noise and statistical CSI," 
IEEE J. Selec. Topics Signal Process., vol. 10, pp. 1462-1477, Aug. 2016.

[31] N. T. Nguyen, H. D. Tuan, T. Q. Duong, and H. V. Poor, "MIMO beamforming for secure and energy-efficient wireless communication," IEEE Signal Process. Lett., vol. 24, pp. 236-239, Feb. 2017.

[32] V. S. Annapureddy and V. V. Veeravalli, "Sum capacity of mimo interference channels in the low interference regime," IEEE Trans. Inf. Theory, vol. 57, pp. 2565-2581, May 2011.

[33] H. H. Kha, H. D. Tuan, and H. H. Nguyen, "Fast global optimal power allocation in wireless networks by local d.c. programming," IEEE Trans. Wireless Commun, vol. 11, pp. 510-515, Feb. 2012.

[34] W. Li, T. Chang, C. Lin, and C. Chi, "Coordinated beamforming for multiuser MISO interference channel under rate outage constraints," IEEE Trans. Signal Process., vol. 61, pp. 1087-1102, Mar. 2013.

[35] W. Li, T. Chang, and C. Chi, "Multi-cell coordinated beamforming with rate outage constraint-part II: efficient approximation algorithms," IEEE Trans. Signal Process., vol. 63, pp. 2763-2778, Jun. 2015.

[36] E. Che, H. D. Tuan, H. H. M. Tam, and H. H. Nguyen, "Successive interference mitigation in multiuser mimo channels," IEEE Trans. Commun., vol. 63, pp. 2185-2199, Jun. 2015.

[37] B. R. Marks and G. P. Wright, "A general inner approximation algorithm for nonconvex mathematical programs," Operation Research, vol. 26, no. 4, pp. 681-683, 1978.

[38] Z. Sheng, H. D. Tuan, A. A. Nasir, T. Q. Duong, and H. V. Poor, "Power allocation for energy efficiency and secrecy of wireless interference networks," IEEE Trans. Wirel. Commun., vol. PP, no. 99, pp. 1-1, 2018.

[39] U. Rashid, H. D. Tuan, H. H. Kha, and H. H. Nguyen, "Joint optimization of source precoding and relay beamforming in wireless MIMO relay networks," IEEE Trans. Commun., vol. 62, pp. 488-499, Feb. 2014.

[40] C. Wang, H. M. Wang, and X. G. Xia, "Hybrid opportunistic relaying and jamming with power allocation for secure cooperative networks," IEEE Trans. Wirel. Commun., vol. 14, pp. 589-605, Feb. 2015.

[41] A. Al-Talabani, Y. Deng, A. Nallanathan, and H. X. Nguyen, "Enhancing secrecy rate in cognitive radio networks via stackelberg game," IEEE Trans. Commun., vol. 64, pp. 4764-4775, Nov. 2016.

[42] T. U. Al-Naffouri, M. Moinuddin, N. Ajeeb, B. Hassibi, and A. Moustakas, "On the distribution of indefinite quadratic forms in Gaussian random variables," IEEE Trans. Commun., vol. 64, pp. 153-165, Jan. 2016.

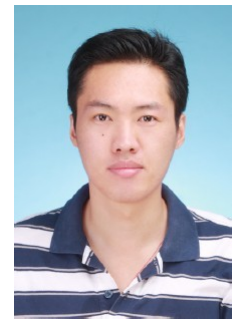

Zhichao Sheng was born in Yangzhou, China. He received the B.S. degree in communication engineering from Nanjing University of Information Science and Technology, Nanjing, China, in 2008, the M.S. degree in signal and information processing from Jiangsu University of Science and Technology, Zhenjiang, China, in 2012. He is currently pursuing the dual-doctoral degree with the University of Technology Sydney, Ultimo, NSW, Australia, and with Shanghai University, Shanghai, China. His research interests include optimization methods for wireless communication and signal processing.

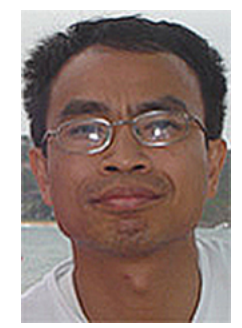

Hoang Duong Tuan received the Diploma (Hons.) and $\mathrm{Ph} . \mathrm{D}$. degrees in applied mathematics from Odessa State University, Ukraine, in 1987 and 1991, respectively. He spent nine academic years in Japan as an Assistant Professor in the Department of Electronic-Mechanical Engineering, Nagoya University, from 1994 to 1999, and then as an Associate Professor in the Department of Electrical and Computer Engineering, Toyota Technological Institute, Nagoya, from 1999 to 2003. He was a Professor with the School of Electrical Engineering and Telecommunications, University of New South Wales, from 2003 to 2011. He is currently a Professor with the School of Electrical and Data Engineering and a Core Member of the Global Big Data Technologies Centre, University of Technology Sydney. He has been involved in research with the areas of optimization, control, signal processing, wireless communication, and biomedical engineering for more than 20 years.

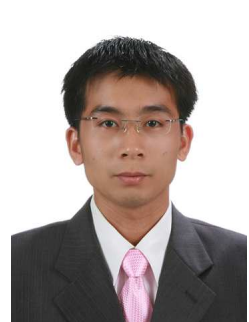

Trung Q. Duong (S'05, M'12, SM'13) received his $\mathrm{Ph} . \mathrm{D}$. degree in Telecommunications Systems from Blekinge Institute of Technology (BTH), Sweden in 2012. Currently, he is with Queen's University Belfast (UK), where he was a Lecturer (Assistant Professor) from 2013 to 2017 and a Reader (Associate Professor) from 2018. His current research interests include Internet of Things (IoT), wireless communications, molecular communications, and signal processing. He is the author or co-author of 290 technical papers published in scientific journals (165 articles) and presented at international conferences (125 papers).

Dr. Duong currently serves as an Editor for the IEEE TRANSACTIONS on Wireless COMMUNiCATIONS, IEEE TRANSACTIONS ON COMMUNICATIONS, IET COMMUNiCATIONS, and a Lead Senior Editor for IEEE COMmunications LetTers. He was awarded the Best Paper Award at the IEEE Vehicular Technology Conference (VTC-Spring) in 2013, IEEE International Conference on Communications (ICC) 2014, IEEE Global Communications Conference (GLOBECOM) 2016, and IEEE Digital Signal Processing Conference (DSP) 2017. He is the recipient of prestigious Royal Academy of Engineering Research Fellowship (2016-2021) and has won a prestigious Newton Prize 2017.

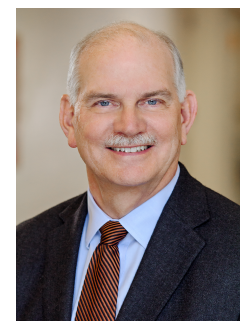

H. Vincent Poor (S'72, M'77, SM'82, F'87) received the Ph.D. degree in EECS from Princeton University in 1977. From 1977 until 1990, he was on the faculty of the University of Illinois at UrbanaChampaign. Since 1990 he has been on the faculty at Princeton, where he is the Michael Henry Strater University Professor of Electrical Engineering. From 2006 until 2016, he served as Dean of Princetons School of Engineering and Applied Science. He has also held visiting appointments at several other institutions, including most recently at Berkeley and Cambridge. His research interests are in the areas of information theory and signal processing, and their applications in wireless networks, energy systems and related fields. Among his publications in these areas is the recent book Information Theoretic Security and Privacy of Information Systems (Cambridge University Press, 2017).

Dr. Poor is a member of the National Academy of Engineering and the National Academy of Sciences, and is a foreign member of the Chinese Academy of Sciences, the Royal Society and other national and international academies. He received the Technical Achievement and Society Awards of the IEEE Signal Processing Society in 2007 and 2011, respectively. Recent recognition of his work includes the 2017 IEEE Alexander Graham Bell Medal, Honorary Professorships from Peking University and Tsinghua University, both conferred in 2017, and a D.Sc. honoris causa from Syracuse University, awarded in 2017. 